Cahiers $d u$ MONDE RUSSE

\section{Cahiers du monde russe}

Russie - Empire russe - Union soviétique et États indépendants

$50 / 1 \mid 2009$

Écrits personnels. Russie XVIII $-\mathrm{XX}^{\mathrm{e}}$ siècles

\title{
Enjeux historiques des journaux et de la correspondance dans la réécriture de l'histoire de la révolution sous Stalin
}

\section{Malte Griesse}

\section{OpenEdition}

Journals

Édition électronique

URL : https://journals.openedition.org/monderusse/9155

DOI : 10.4000/monderusse. 9155

ISSN : $1777-5388$

Éditeur

Éditions de l'EHESS

Édition imprimée

Date de publication : 31 mars 2009

Pagination : 93-124

ISBN : 978-2-7132-2259-7

ISSN : $1252-6576$

Référence électronique

Malte Griesse, «Enjeux historiques des journaux et de la correspondance dans la réécriture de I'histoire de la révolution sous Stalin », Cahiers du monde russe [En ligne], 50/1 | 2009, mis en ligne le 01 janvier 2009, consulté le 02 septembre 2022. URL : http://journals.openedition.org/monderusse/ 9155 ; DOl : https://doi.org/10.4000/monderusse.9155 


\section{ENJEUX HISTORIQUES DES JOURNAUX ET DE LA CORRESPONDANCE DANS LA RÉÉCRITURE DE L'HISTOIRE DE LA RÉVOLUTION SOUS STALIN ${ }^{1}$}

On a longtemps pensé que « stalinisme » et « journaux intimes » étaient incompatibles. S'il était presque inimaginable pour les Occidentaux que l'on ait pu, sous un tel régime, coucher sur le papier ses pensées secrètes (et bien entendu hérétiques) et risquer ainsi d'être découvert, dénoncé et réprimé, il était plus inconcevable encore que l'on ait pu tenir un journal pour exprimer son enthousiasme, sauf à supposer que l'on affichait ainsi sa loyauté afin d'éviter d'être arrêté. Aussi les publications soviétiques de récits personnels ne furent-elles pas prises au sérieux et furent longtemps mises au rang d'une pure et simple propagande. C'est donc le grand mérite de la recherche récente sur la subjectivité que d'avoir rectifié cette tenace idée d'un for intérieur inaltérable, insensible à la conjoncture historique qui, certes, pouvait être brisé par un État totalitaire, mais en aucun cas devenir ce que proclamaient les idéologues soviétiques dans leur mot d'ordre de la création de l'Homme nouveau. Pour la première fois, on s'autorisait à penser que les individus pouvaient à l'époque stalinienne se prononcer et agir en accord avec les préceptes du régime non par cynisme, carriérisme ou peur, mais par profonde conviction ${ }^{2}$.

1. Je remercie vivement André Berelowitch, Alain Blum, Yves Cohen, Elisabeth Teiro, Gesine Müller, Viktor Sorokatyj, ainsi que Jutta Scherrer et les participants à son séminaire à l'EHESS, pour les échanges précieux sur des questions abordées dans le présent texte. Je dois beaucoup à Laurent Thévenot, tant à son séminaire à l'EHESS sur les « liens du proche au public », qui sont au cœur de mon investigation, qu'à nos nombreux échanges au sujet de mon travail. J'ai beaucoup tiré profit de ses remarques et ses critiques. Son esprit ouvert, sans jamais entraver ma liberté, m'a résolument encouragé à trouver mon propre chemin intellectuel, mes propres façons d'aborder les questions.

2. Cf. l'aperçu général sur les conceptions implicites du sujet dans les études sur le stalinisme : Anna Krylova, « The Tenacious Liberal Subject in Soviet Studies », Kritika : Explorations in Russian and Eurasian History, 1 (1), Winter 2000, p. 119-146. 
Le changement radical de perspective de cette dernière décennie résulte des nouvelles découvertes de documents et de l'impact croissant de la théorie du discours sur les sciences humaines qui a fini par investir le domaine des études russes $^{3}$. Désormais, on tend de plus en plus à envisager les diverses pratiques staliniennes sous un angle nouveau : elles ne sont plus vues comme de pures contraintes au profit d'objectifs extérieurs qui n'auraient rien en commun avec les besoins et soucis de la population, mais comme des « offres identitaires » permettant aux individus d'embrasser les grands objectifs du projet bolchevik. L'autocritique, les déclamations publiques de biographies, l'engagement politique et le «travail social »(obščestvennaja rabota), au même titre que l'émulation socialiste, le stakhanovisme, et même le travail forcé en camps acquièrent dans cette optique une nouvelle dimension. Ce ne sont plus tentatives d'effacer la personne mais un très large éventail de procédés destinés à émanciper les masses populaires du principal fléau russe - l'arriération - et à doter les individus (ouvriers et paysans) d'une voix (publique) et, par là, d'une nouvelle dignité, qui consistait précisément à s'approprier ces grands objectifs de l'humanité mis en avant par le régime stalinien.

Or, effectivement, les journaux intimes, exhumés d'archives les plus diverses, tiennent souvent un tout autre langage que celui attendu, ne serait-ce que parce qu'ils parlent avant tout ${ }^{4}$. Dans ces récits, plus ou moins fragmentés selon les genres, les stéréotypes du langage officiel ne manquent pas, mais ils sont si intimement mêlés au vécu personnel qu'ils en deviennent moyens d'expression essentiels des auteurs. Ceux-ci, dans la plupart des cas, se situent à l'intérieur du « système », non en dehors. Non plus simple façade, affichée uniquement pour répondre aux attentes « d'en haut », cette « langue de bois » semble donc avoir pénétré la perception la plus intime et façonner ainsi le réel, cet univers que les gens habitaient. D'où l'exigence de porter plus d'attention à la représentation du système qu'au réel, voire à prendre le « discours » pour le « réel $»^{5}$.

De nombreux journaux intimes ont montré à quel point être « à la hauteur » du processus historique était considéré comme une obligation et un acquis moral, combien il était nécessaire d'appartenir au parti bolchevik non seulement formellement, mais aussi au sens intrinsèque, même si (et souvent parce que) la participation à la construction de l'ordre communiste final imposait d'innombrables sacrifices personnels. Aussi les diaristes faisaient-ils des efforts considérables pour s'inscrire dans les grands objectifs de la construction du communisme, du parachèvement de l'histoire, pour dépasser la «petitesse » ou la «mesquinerie » d'une

3. Ce tournant a été largement mis en avant par Stephen Kotkin qui, le premier, envisage le stalinisme comme «civilisation », s'appuyant essentiellement sur la théorie foucaldienne du discours (Magnetic Mountain : Stalinism as a Civilization, Berkeley-Los Angeles-Londres : University of California Press, 1995).

4. Voir les journaux publiés : Véronique Garros, Natalia Korenevskaya, Thomas Lahusen,éds., Intimacy and Terror: Soviet Diaries of the 1930s, New York: The New Press, 1995 ; Jochen Hellbeck, éd., Tagebuch aus Moskau 1931-1939, Munich : Deutscher Taschenbuch Verlag, 1996.

5. Cf. le chapitre « Speaking Bolshevik » in Kotkin, Magnetic Mountain, p. 198-238. 
existence purement privée : privée en fait des choses essentielles et grandes qui n'étaient envisageables qu'au niveau du collectif ${ }^{6}$. Ces écritures personnelles témoignent donc de l'ardeur héroïque et de l'exaltation du soi qu'a engendrée l'identification avec le tout et l'appropriation d'objectifs universels ${ }^{7}$. Bref, ces journaux, « laboratoires du soi stalinien $»^{8}$, ont largement contribué à rendre plus intelligible la profondeur de l'impact du stalinisme sur les consciences des citoyens soviétiques.

Un argument fort milite en faveur de la sincérité de ces textes. On trouve des doutes exprimés çà et là dans le récit, que ce soit à propos d'une mesure politique, de la condamnation d'un «ennemi du peuple» (généralement de l'entourage personnel) ou même de la ligne générale dessinée par Stalin. D’après ce que nous savons de la « logique » de la persécution à l'époque, la découverte d'un document contenant de tels doutes suffisait à justifier (amplement) l'arrestation de son auteur ${ }^{9}$. Il faut donc prendre d'autant plus au sérieux les crises provoquées par les doutes, qui s'expriment parfois dès les pages suivantes : c'est en se relisant que les diaristes commencent à se fustiger et cherchent à dissiper leurs doutes. Remarquons une caractéristique des passages ou épisodes les plus frappants dans ce sens c'est-à-dire ceux où l'identité personnelle du diariste est à tel point liée au système que tout doute quant à la justesse de la ligne politique du moment entraîne chez lui une grave remise en question de soi - : ils résultent pratiquement tous d'un isolement profond de l'entourage immédiat, d'une rupture des liens personnels, d'un manque d'échange franc avec les proches. C'est du moins l'hypothèse que permet d'avancer la perception des auteurs eux-mêmes ${ }^{10}$.

\footnotetext{
6. Voir déjà chez Marx l'image du grand esprit et génie individuel qui devient pitoyable dans ses possibilités d'action face à la classe consciente qui agit en commun. Karl Marx, Theorien über den Mehrwert III, vol. 26, p. 307, in Karl Marx/Friedrich Engels - Werke, Berlin : (Karl) Dietz Verlag.

7. Pour la mise en parallèle de « transformation économique » et « transformation personnelle », cf. le journal de Marietta Šaginjan, Dnevniki 1917-1931,L., 1932, p. 7-8, qui se présente comme un modèle pour le lecteur soviétique.

8. Cf. Jochen Hellbeck, Revolution on My Mind: Writing a Diary Under Stalin, Cambridge, MA : Harvard University Press, 2006.

9. En témoignent les journaux (peu nombreux) qui ont pu être retrouvés dans les archives du FSB avec les soulignements des enquêteurs. Voir le journal du paysan Aržilovskij, in Intimicy and Terror, celui de Nina Lougovskaia, Journal d'une écolière soviétique (P.: Pocket Jeunesse, 2007), et celui du «national bolchevik» Vasilij Kuraev, Dnevnik ssyl'nogo bol'ševika 19331935 [Journal d'un bolchevik déporté] in V.V. Kuraev, G.V. Lebedev, M.S. Polubojarov, M. : Azbuka, 1997.

10. Cf. Ioulia Piatnitskaja, Chronique d'une déraison. Moscou 1937-1938. Journal annoté par Igor Piatnitski, P. : Seuil, 1992; Jochen Hellbeck, Tagebuch aus Moskau 1931-1939; Aleksandr Afinogenov, «Dnevnik 1937 goda », Sovremennaja dramaturgija, n 1, 1993, p. 219-233, n² 2, p. 223-241, n 3, p. 217-239. Pjatnickaja commence à tenir son journal (sa « chronique d'une déraison ») immédiatement après l'arrestation de son mari qui était auparavant pour elle l'incarnation du parti et de la cause communiste. Tout son monde et le réseau d'amis et de connaissances s'écroulent presque immédiatement. Chez Afinogenov, dramaturge très populaire de la première moitié des années 1930, le journal, d'un outil de sa production littéraire qu'il était, change radicalement de caractère après son exclusion du parti et l'isolement qui s'ensuit : il
} 
C'est le cas de Stepan Podlubnyj ${ }^{11}$. Ce fils d'un paysan dékoulakisé arrive à Moscou en 1931 sous une fausse identité. Il cherche, à travers son travail et son journal intime, à s'intégrer dans le système de valeurs stalinien afin d'exorciser ses origines sociales «impures ». Il n'en exprime pas moins à plusieurs reprises des doutes sur la politique stalinienne. Mais il dénonce aussitôt son hérésie qu'il attribue à son essence d'ennemi de classe qui, loin d'être apurée, lui impose en permanence un grand travail sur lui-même. Obligé de dissimuler son identité sociale même à ses amis, il déplore son isolement et voit dans son journal son seul « ami intime », et pourtant aussi comme la décharge de toutes les «ordures » que sont ses pensées hérétiques et « illégitimes».

Bien que le journal ne manque ni d'hésitations ni de vacillations, c'est pratiquement toujours au moment de rapprochements et de communication ouverte avec ses amis que ses doutes se cristallisent en critique résolue. Ainsi, au tout début de l'année 1935, il soupçonne le régime d'être le vrai responsable du meurtre de Kirov (comme d'ailleurs de la mort soudaine de Kujbyšev peu après) et de s'en servir pour justifier une vague de répressions. Il la compare à la réaction à la révolution de 1905 sous Stolypin, qu'il connaît par le manuel d'histoire du parti (12.02.1935). Cette analyse extrêmement critique, univoque et somme toute lucide, va de pair avec des échanges particulièrement intenses et ouverts dans le cercle de ses amis avec qui il finit même par projeter la rédaction d'un journal clandestin qui traiterait de sujets sensibles passés sous silence dans la presse officielle (18.02.1935). Plus tard (début 1936), lorsqu'il est « démasqué » au sein du Komsomol, il apparaît qu'il y a encore autour de lui un certain nombre de jeunes gens de même origine sociale et qui, finalement, s'en sont ouvert les uns aux autres.

Même si ses problèmes (son exclusion du Komsomol, la mise en place de la passeportisation qui le place sous la menace d'une expulsion de la capitale, etc.) sont loin d'être résolus, Podlubnyj exprime son grand soulagement de pouvoir enfin parler franchement avec ses amis. Il constate que tous les «ci-devant» (byvšie) de son entourage sont en fait parmi les meilleurs des travailleurs, des héros du travail, des gens admirables. Pourtant le régime continue à mener à leur égard une politique discriminatoire, malgré des démentis dans la presse et même, peu après, dans la Constitution stalinienne. Dans son journal, Podlubnyj se limite à une seule allusion : ce traitement des «éléments socialement étrangers » permet de «tirer des conclusions intéressantes» sur la nature du régime (21.02.1936). D'ailleurs, « son amour du journal » se refroidit rapidement, il y écrit de moins en

devient un moyen ou plutôt le moyen principal d'un travail sur soi, de l'intériorisation de la ligne du parti. Tous ces auteurs déplorent à maintes reprises leur solitude, de ne pas pouvoir parler librement à leur entourage. Cf. les travaux de Jochen Hellbeck, particulièrement son introduction à Tagebuch aus Moskau et «Writing the Self in the Time of Terror : Alexander Afinogenov's Diary of 1937 », in Laura Engelstein, Stephanie Sandler, éds., Self and Story in Russian History, Ithaca-Londres : Cornell University Press, 2000, p. 69-93.

11. Les lignes suivantes s'appuient sur la publication en allemand du journal de Stepan Podlubnyj par Jochen Hellbeck, Tagebuch. Il s'agit là d'un témoignage fascinant qui eut un énorme impact dans le monde de la recherche germanophone. Hélas, ce journal, conservé aux Archives du peuple (Centr dokumentacii Narodnyj Arhiv), n'est actuellement accessible qu'en cette langue. 
moins. Il peut désormais discuter avec ses amis de tout, y compris des taches de sa biographie, dont l'occultation était jusque-là le motif essentiel de son écriture diariste (18.06.1936).

La correspondance qu'il entreprend avec des camarades d'enfance (10.05.1936), puis avec son meilleur ami lorsque celui-ci est appelé au service militaire (23.11.1936), semble rendre de plus en plus superflu un journal où il n'aurait presque plus rien à dire sans risquer de se répéter. Podlubnyj écrit de manière plus irrégulière et le monologue du journal y cède de plus en plus souvent la place au dialogue, y compris à celui de la correspondance, du moins en ce qui concerne la dimension existentielle de son auteur ${ }^{12}$. Abstraction faite des nombreuses autres fonctions, le journal est lieu de travail sur soi. Sous cet angle, c'est le repaire où l'auteur ne se réfugie qu'en cas d'isolement extrême pour pallier un manque existentiel de communication ouverte dans le cadre d'une proximité qu'il juge intuitivement propre à mieux résoudre les problèmes qui le tourmentent.

Les hésitations entre doute et rétractation, la difficulté manifeste à se positionner et la fragilité de l'identité personnelle (même entendue comme identité réformable) semblent refléter les contradictions extérieures, sociétales et systémiques, qui sont ainsi intériorisées et transformées en contradictions du for intérieur. L'identité personnelle, c'est-à-dire la perception de soi, n'est rapidement stabilisée que lorsque le diariste, par le biais d'une meilleure qualité de la communication, surtout des échanges amicaux, arrive à surmonter le décalage entre l'engagement affiché devant l'entourage et les soucis secrets qui ont jusqu'alors dominé ses réflexions solitaires. Dissimuler ses préoccupations réelles l'a rendu hypocrite : il est conscient de ne montrer qu'un faux visage mais, avec le temps, c'est son « vrai » visage qu'il ne reconnaît plus, il ne sait plus qui il est, notamment parce qu'il a fait sienne l'idée moderne de personne comme projet, d'identité malléable. Ce n'est qu'au travers de la perception d'autrui qu'il se réapproprie une identité cohérente, ce qui n'obère pas pour autant l'idée de la nécessité du progrès et du développement individuels. Mais c'est la communication ouverte et la réciprocité de la perception qui font que la stagnation qu'il observe parfois dans son propre développement est dissociée de la souillure de son origine sociale. Celle-ci, perdant de son acuité, est

12. La correspondance tardive et, d'après ce que Podlubnyj affirme, plus ouverte, n'est malheureusement pas gardée. Cf. Hellbeck : Tagebuch, et « Fashioning the Stalinist Soul. The Diary of Stepan Podlubnyi (1931-1939) », Jahrbücher für Geschichte Osteuropas, Neue Folge, 44, 3, 1996, p. 344-373 pour la publication allemande et une interprétation passionnante qui met essentiellement en relief l'aspect de la dépendance discursive et identitaire envers le système. Hellbeck s'intéresse aussi à la communication : il y voit en premier lieu un moment stabilisateur pour l'identité stalinienne. À partir d'un échange de lettres entre Podlubnyj et son cousin — lui aussi fils de dékoulakisé — , il montre comment Podlubnyj se présente en bon stalinien, au point de prétendre bien réussir ses études (tandis que le journal dit le contraire) et de majorer même son salaire tout en passant sous silence les énormes difficultés matérielles qu'ils ont à affronter. En visite dans son village natal, Podlubnyj se présente comme un intellectuel accompli et, pour bien l'afficher, il s'affuble même de fausses lunettes ! Or il avait apparemment des comptes à régler avec ceux qui étaient restés et qui avaient appliqué la dékoulakisation. Tout cela semble illustrer surtout le degré d'aliénation entre Podlubnyj et ceux auxquels il avait affaire, d'autant plus qu'il voyait lui-même les choses de cette manière. 
perçue comme l'entrave purement extérieure d'une politique discriminatoire. D'où aussi le tournant critique de ses réflexions.

Certes, la crise d'identité engendrée par l'isolement du sujet est le revers de la médaille de l'individu moderne en général, mais ne s'agit-il pas ici d'un idéal-type de l'individu atomisé comme terrain particulièrement propice à la pensée totalitaire, telle que Hannah Arendt, dans son étude classique sur le totalitarisme, l'a formulée ? La projection des doutes sur le moi propre et la recherche d'un appui stable dans la «logique déductive » de l'idéologie ne sont-elles pas le fruit de l'ambition totalitaire du régime stalinien ${ }^{13}$ ? On ne peut le nier, puisque toutes les pratiques répressives de l'époque stalinienne visent non pas directement la personne, qui doit être éduquée (quand il y a encore de l'espoir) ou écrasée (quand il s'agit d'un contre-révolutionnaire invétéré) ${ }^{14}$, mais en tout premier lieu les liens interpersonnels. Que ce soit devant les commissions de contrôle, au cours des interrogatoires en prison ou lors des grands procès-spectacles, partout les questions posées concernent essentiellement les liens (svjazi).

Voyons de plus près les liens personnels et les échanges communicationnels dans les cadres de proximité ${ }^{15}$, c'est-à-dire hors des espaces directement contrôlés et enregistrés dans les sténogrammes et les procès-verbaux. Le journal de Podlubnyj laisse entrevoir à quel point il est malaisé pour un individu isolé de se positionner (fermement), mais aussi combien l'état d'isolement fragilise. Il montre la rapidité avec laquelle les doutes peuvent se retourner contre le régime, lorsque les liens de communication avec l'environnement immédiat sont rétablis ou (re)créés. Pourtant, nous observons aussi que tout ce qui concerne l'interaction avec l'environnement, la communication, etc., se passe essentiellement en dehors du cadre introspectif du journal et n'y est abordé que de façon indirecte, avec le recul de la réflexion, qui cherche à (r)établir de la cohérence ${ }^{16}$. Nous envisageons donc la constitution de la personne non seulement dans la pratique solitaire,

13. Hannah Arendt, Elemente und Ursprünge totaler Herrschaft: Antisemitismus, Imperialismus, totale Herrschaft, Munich-Zurich: Piper, 20039', p.971-979 (trad. française, Les origines du totalitarisme, P. : Seuil, 1998). Pour la dissociation des individus dans les sociétés modernes, cf. Hannah Arendt, Condition de l'homme moderne (trad. de l'anglais, P. : Presses Pocket, 1993).

14. Igal Halfin (Intimate Ennemies: Demonizing the Bolshevik Opposition, 1918-1928, Pittsburgh : University of Pittsburgh Press, 2007) voit dans les pratiques staliniennes surtout une tentative d'éduquer les individus. Pour cette raison, il parle d'une « herméneutique des âmes ».

15. Pour « cadres de proximités », nous nous appuyons sur la différenciation et l'étagement des liens du proche jusqu'au public, tels que les a élaborés Laurent Thévenot ( $L$ 'action au pluriel : les régimes d'engagement, P. : La Découverte, 2006). Il distingue différents « régimes de proximité » pour dégager les différentes formes d'engagement. Voir aussi le Programme du $\mathrm{CNRS}$ « Des liens du proche au public » dirigé par Laurent Thévenot et Daniil Aleksandrov. Le système soviétique fait tout pour contrôler les rapports des citoyens jusque dans la sphère privée, la question du lieu de ses sociabilités y revêt alors une importance particulière. Voilà pourquoi je parle de « cadres » de proximité.

16. Sur la cohérence narrative et sa dimension éthique, cf. Paul Ricœur, Soi-même comme un autre, P. : Seuil, 1990. 
introspective (souvent face au pouvoir), telle qu'elle s'exprime dans un journal, mais aussi dans l'interaction, dans les échanges intersubjectifs à tous les niveaux.

Pour confirmer notre hypothèse, analysons quelques échantillons de la communication familiale que nous extrayons de la correspondance très riche d'Anna Grigor'evna Kravčenko (1890-1984) et d'Aleksandr Petrovič Spunde (1892-1962). Ces vieux bolcheviks se sont connus au printemps 1917 à Perm. Ils y travaillaient pour le parti, avec leur fils Jakov, né en 191817. Révolutionnaires bolcheviks engagés depuis l'âge de dix-sept ans, Kravčenko et Spunde occupent rapidement après la révolution d'Octobre des postes de responsabilité dans le jeune État soviétique. Spunde, né dans une famille d'ouvriers en Lettonie, a été exilé pendant quatre ans (de 1913 jusqu'à la révolution de Février 1917) dans le gouvernement de l'Enisej. Après la révolution, il travaille surtout dans le domaine des finances (pour la banque d'État et le commissariat du peuple aux Finances, Narkomfin) puis, à partir des années 1930, pour le commissariat du peuple aux Transports (NKPS). En 1932, il se voit obligé de prendre sa retraite pour raisons de santé (il souffre d'épilepsie depuis 1927)18. Au début de l'année 1918, il rejoint brièvement les communistes de gauche menés par Buharin, qui s'est opposé à la conclusion de la paix de Brest-Litovsk. Ce sera l'un des chefs d'inculpation lorsque Spunde est exclu du parti en mars 1938, lors du deuxième grand procès contre le « bloc antisoviétique de droitiers et trotskistes ». Ce groupe d'opposition y est, rétrospectivement, accusé d'avoir planifié en 1918, en collaboration avec les sociauxrévolutionnaires, un attentat contre Lenin, Sverdlov et Stalin ${ }^{19}$.

17. Le fonds de la famille est conservé au GARF (Gosudarstvennyj arhiv Rossijskoj federacii - Archives d'État de la Fédération de Russie), f. R-9665, op. 1. Rien qu'entre les deux époux, environ 1200 lettres couvrent toute la période allant de la révolution à la mort de Spunde en 1962 (d. 67-79). Une partie de ces lettres, surtout celles des premières années du régime soviétique, ont été publiées : I.B. Brajnin, éd. « Samo prošedšee, kak ono bylo... » perepiska Anny Kravčenko i Aleksandra Spunde [«Le passé tel qu'il était... » correspondance d'Anna Kravčenko et d'Aleksandr Spunde], M. : Izdat. polit. literatury, 1990. À part la correspondance - qui ne se limite pas au seul cercle de la famille, même si ce matériau est le plus riche -, le fonds contient de nombreux manuscrits de Mémoires (vospominanija) de Kravčenko (d. 52-55, 58, 60-61, 63) et surtout de Spunde (d. 201-208), rédigés à partir de la Seconde Guerre mondiale. À partir de cette époque, Spunde travaille intensément sur l'histoire russe et recopie des extraits de ses lectures (d. 199-200,218-222) et de nombreux manuscrits (d. 213-217) qui ont le caractère de monographies. Kravčenko a tenu un journal au moins à partir de la révolution, mais les carnets ne sont conservés que depuis 1937. Il s'agit aussi bien d'un journal de travail que d'un journal "privé ». Bien que les deux journaux ne soient pas introspectifs et ressemblent plutôt à des blocs-notes, ils se révèlent parfois précieux dans notre perspective puisque nous y trouvons des notes (souvent très brèves) sur des entretiens qu'elle a eus, ou quand elle y recopie soigneusement des lettres reçues.

18. Cf. les autobiographies de Spunde (GARF, f. R-9665, op.1, d. 176) ; les esquisses biographiques de Jakov sur son père (ibid., d. 197, 1. 52-55, d. 212 passim) ; les préfaces des manuscrits historiographiques de Spunde rédigés par Jakov après la mort de son père (d. 213 et 214).

19. Cf. les lettres de justification de Spunde aux dirigeants du parti après son exclusion, GARF, f. R-9665, op. 1, d. 228. Pour les accusations contre les communistes de gauche, cf. Sudebnyj otčet po delu antisovetskogo «Pravo-trockistskogo bloka » rassmotrennomu voennoj kollegiej verhovnogo suda SSSR. 2-13 marta 1938 po obvineniju... Polnyj tekst stenografičeskogo otčeta, [Compte rendu du procès sur l'affaire du «bloc de droite trotskiste » antisoviétique jugée par le collège militaire de la cour suprême d'URSS. 2-13 mais 1938, sous l'inculpation de..., Texte complet du compte rendu sténographié], M. : Izdanie narodnogo komissariata justicii SSSR, 1938. 
Kravčenko, pédagogue engagée en Sibérie à l'issue de la guerre civile, est dès 1920 «découverte » et recrutée pour travailler au commissariat du peuple à l'Instruction (Narkompros) par Nadežda Krupskaja dont elle devient l'adjointe à la tête de l'Administration principale pour l'Instruction politique (Glavpolitprosvet). Elle travaille comme rédactrice pour plusieurs journaux, surtout dans le domaine de la «liquidation de l'analphabétisme » (likvidacija bezgramotnosti, likbez). En 1937, à la suite d'une dénonciation anonyme, elle est réprimandée pour « myopie » et «manque de vigilance » face aux « ennemis du peuple» : son journal paysan pour lecteurs débutants (Krest’janskaja gazeta dlja načinajuščihsja čitat') aurait condamné les accusés des procès trotskistes de 1936 et 1937 en termes trop modérés et même cité Trockij avec ses initiales ${ }^{20}$. À peu près en même temps, leur fils Jakov, accusé d'avoir gardé le contact avec un ancien professeur d'histoire arrêté comme « ennemi du peuple », est exclu du Komsomol. Néanmoins, toute la famille survit aux répressions staliniennes et Spunde est réhabilité et réintégré au sein du parti après le XXe Congrès en 1956.

Analysons à présent l'échange intensif dans le cadre de la famille, centré autour d'Aleksandr Spunde, tel qu'il peut être appréhendé dans la correspondance familiale. Notre objectif est de montrer à quel point un cadre de communication ouverte engendre ses propres façons de voir et de comprendre, ses propres modèles interprétatifs, ses propres « discours » qui sont en rapport avec « le discours ambiant», mais pas toujours en consonance avec lui. Tout au contraire, ils prennent un tour extrêmement critique qui dépasse largement l'expression d'un vague mécontentement. Pour résumer de façon très simpliste et schématique le contenu des pages qui suivent, nous allons tenter de montrer comment la communication engagée aide à se situer à l'intérieur du système, pour ensuite illustrer comment elle rend possible une prise de position résolument opposée à ce même système, tout en cherchant des possibilités de trouver une praxis appropriée à la théorie.

\section{Communication et affirmation}

Durant les années 1920, Spunde et Kravčenko soutiennent la politique de Stalin contre les oppositions de gauche et de droite : plusieurs prises de position en faveur de la politique de Stalin peuvent être décelées dans leur correspondance. Il s'en dégage l'impression que leur échange intense y joue un rôle non négligeable. Cependant, contrairement à l'époque suivante des années 1930-1950, lorsque le régime stalinien est déjà fermement installé et toute controverse politique quasiment bannie de la sphère publique, la correspondance permet également d'entrevoir l'impact des échanges avec d'autres acteurs sur les prises de décision.

L'un des objectifs significatifs le plus fréquemment rencontrés dans la correspondance est ce besoin de faire part de ses impressions, de discuter avec l'autre sur 
l'évaluation d'une situation ou d'une mesure politique, etc. («hočetsja podelit'sja... »). La façon et la fréquence avec lesquelles Spunde exprime ce besoin montrent combien cette activité est pour lui vitale. Naturellement, ce n'est que dans les périodes de séparation, lorsqu'au moins un des interlocuteurs est absent (surtout en mission), qu'ils sont obligés de satisfaire leur besoin de communiquer par courrier.

Avec une impatience aiguë j'attends de te voir. J'ai traversé beaucoup de choses et j'ai extrêmement besoin de t'en faire part (7.04.1918). ${ }^{21}$

Soulignons combien cette communication, loin d'être unilatérale, est un échange réel d'opinions, dont la dynamique propre incite chaque interlocuteur à changer de perspective, modifier son point de vue, envisager autrement les sujets discutés. Même si les échanges aboutissent à une décision commune (ce n'est pas toujours le cas), chacun élabore, au cours de la discussion, sa propre perspective, son propre point de vue. Nous constatons généralement combien cet échange d'opinions rend les interlocuteurs plus sûrs d'eux-mêmes, les renforce dans leur jugement même.

Notons les hésitations de Spunde face à l'émergence de l'opposition de droite : il approuve l'industrialisation accélérée mais est plus réservé en ce qui concerne la réalisation de cette industrialisation, sur le fait qu'elle se fasse aux dépens de la paysannerie. On découvre ses entretiens personnels avec les principaux acteurs de la droite, avec Buharin et Rykov (avec qui il est en très bons termes), mais aussi avec Frumkin (pour lequel il nourrit une antipathie datant déjà de son exil prérévolutionnaire, auquel remonte leur conflit) - et tout cela par le prisme de la correspondance avec sa femme qui se trouve alors aux États-Unis, en mission pour le Narkompros. En dépit d'omissions volontaires, ils abordent largement la controverse au sein du parti, avec un code peu difficile à déchiffrer ${ }^{22}$, sans tomber toujours

21. Ici la question de la censure surgit. Les correspondants sont-ils conscients qu'un tiers puisse les lire ? Ne faut-il pas supposer un écart important entre ce qui est communiqué par courrier et ce qui l'est de vive voix ? En général, la correspondance de toute la décennie 1920 ne révèle pas de « lecteur implicite ». Pratiquement tous les thèmes sont abordés, même si on rencontre aussi parfois des commentaires du genre «nous en parlerons de vive voix » (ob etom pri vstreče). D'ailleurs, très souvent, évitant la poste, les deux correspondants accumulent leurs lettres en attendant qu'un camarade ou une connaissance fasse le trajet pour les transmettre en mains propres (s okaziej). Souvent ils mentionnent cette personne et de la façon dont ils en parlent, on peut entrevoir les rapports qui les lient. Ainsi, par moments, ils renvoient un sujet à une lettre ultérieure qu'ils espèrent pouvoir faire parvenir par l'intermédiaire d'une personne plus proche, en qui ils ont plus confiance. Cette pratique n'est très probablement pas abandonnée dans les années 1930, même si on a l'impression qu'il y a davantage de sujets évités, ce qui n'empêche pas que ce que l'on évoque découle d'un véritable besoin d'échange. En général, par rapport aux premières années du régime soviétique, où la politique intérieure et extérieure sont largement évoquées, on peut déceler un déplacement d'accent vers la politique étrangère, surtout à partir de la guerre d'Espagne : plusieurs amis étrangers (un Espagnol, un couple américain, un Japonais) sont volontaires dans les Brigades internationales. Néanmoins, dès 1934, on trouve, même si c'est rare, des commentaires comme «Pour le moment, écris sur les petits oiseaux. Comme moi dans cette lettre où je n'évoque en rien la politique. » («Piši o ptičkah poka. Ja svoe piśmo tože sdelala i bez politiki », lettre de Kravčenko à Spunde, 17.04.34).

22. Souvent ils recourent à des abréviations : «la controverse entre S. et R.» (Stalin et Rykov) ; « Aujourd'hui M.I.F. est venu me voir (celui chez qui tu étais juste avant ton départ) » (M.I. Frumkin), etc. 
d'accord sur le sens des événements politiques en URSS. De Californie, Kravčenko les suit avec un certain retard (qu'aggravent les délais de la poste) à travers la presse soviétique accessible chez son hôte, une communiste américaine. Malgré sa difficulté à «séparer sympathie personnelle et sympathie politique» (23.12.1928), Spunde finit par se ranger à la ligne politique de Stalin. À en juger par la correspondance, il cède surtout sous l'effet de cette supériorité technologique américaine que sa femme décrit de façon si impressionnante dans ses lettres. Ce sont le niveau économique et organisationnel (que Kravčenko lui décrit à l'aide de schémas du fonctionnement d'entreprises qu'elle a visitées), ainsi que le niveau américain de l'équipement militaire (dont l'impact est d'autant plus grand que les relations avec le monde capitaliste sont tendues depuis le refroidissement diplomatique entre l'URSS et la Grande-Bretagne), qui contribuent largement à persuader Spunde de l'urgence de « rattraper et dépasser [les pays capitalistes] » et de l'impossibilité de se contenter de la croissance modérée de la NEP.

Pendant toute la révolution culturelle (au moins jusqu'à la retraite de Spunde en 1932), les deux correspondants continuent envers et contre tout à soutenir la collectivisation avec enthousiasme. Ils sont toujours obsédés par la nécessité d'atteindre le niveau technologique des États-Unis pour contrer la menace d'une guerre jugée imminente. À plusieurs reprises, ils se réfèrent à leurs entretiens au sujet de l'expérience américaine, notamment en ce qui concerne les préparatifs des pays capitalistes à la guerre :

Et de nouveau, il y a des chances de repousser la guerre ne serait-ce que jusqu'au début du Deuxième Plan quinquennal. [...] Nous avons déjà collectivisé $40 \%$ de l'agriculture. Avant le début des semailles au nord nous arriverons probablement à $45 \%$ et en automne on peut compter atteindre les $60 \%$ ou même plus. Tu te souviens de nos entretiens sur la guerre ${ }^{23}$. Et alors, il n'est pas exclu qu'elle éclate à un moment où notre chance de remporter la victoire ne dépendra plus uniquement du développement de la lutte des classes chez l'adversaire, mais reposera déjà sur notre supériorité ou du moins sur un niveau technologique égal chez nous. (Spunde à Kravčenko 29.03.1931)

Cela ne les empêche cependant pas de mettre en cause les « excès » (peregiby) de la collectivisation forcée et cela visiblement dès avant la publication de la fameuse lettre de Stalin du 2 mars 1930 sur les vertiges du succès. En même temps, Spunde laisse entrevoir son indignation quant à la manière stéréotypée de rejeter la faute sur des boucs émissaires locaux : «il en ressort qu'à Moscou tous sont bien gentils mais que sur place [il n'y a que des] méchants ». (11.02.1930)

L'espoir suscité par le Deuxième Plan quinquennal est cependant lié à l'accent qu'il met sur une amélioration des conditions de vie de la population, qui devrait résulter de la baisse des subventions unilatérales pour l'industrie lourde caractéristiques du Premier Plan.

23. Pour les mots en italiques, c'est nous, M.G., qui soulignons ; les mots soulignés le sont dans l'original. 
Voilà, ce [deuxième] plan quinquennal ne sera plus d'un genre purement industriel, mais il met l'accent aussi sur les conditions de vie - sur le ravitaillement, sur la distribution (raspredelenie) de vêtements, sur la résolution de la pénurie de logements, une énorme croissance de la base pour une socialisation du mode de vie, - une mécanisation du nettoyage des locaux, de la préparation de repas... $(23.03 .1931)$

Comme chez beaucoup d'autres citoyens soviétiques, notamment chez ceux qui avaient du mal à supporter les inégalités sociales de la NEP, l'élan révolutionnaire est renouvelé par le « grand pas en avant ». Mais le présent en soi n'est jamais considéré comme satisfaisant, l'enthousiasme se fonde toujours sur la perspective (l'illusion ?) d'un avenir meilleur qui reste la seule justification aux sacrifices du présent. Cela est particulièrement évident lorsqu'on regarde la réalité soviétique avec les yeux d'un étranger. Quand une amie américaine, communiste et sympathisante du jeune État soviétique, venue vivre en Union soviétique, affronte de nombreuses difficultés, Spunde anticipe déjà son éventuel désenchantement, se met à sa place pour faire le bilan sans concession de «notre vie », pour tout de suite justifier ce mauvais présent en pensant au futur, usant de tous les sophismes de la dialectique :

11.04.1931... À propos de Harriet. Je pense qu'il ne serait pas très raisonnable d'essayer de la retenir. Pour son âge, notre vie est assez rude, à ce qu'il paraît. Qu'elle se retourne contre nous aux États-Unis par désenchantement, je ne crois pas. En même temps, il ne faut pas se faire d'illusions. Quand on brise toute une vie aussi brutalement, et qu'en plus, c'est souvent le fait de gens fourbus et irritables, les ennuis et les accrocs ne sont pas du tout un hasard. Quand on réfléchit de façon dialectique, cela n'est qu'un symptôme de ce que nous refaisons la vie - il y a des signes de résistance, [mot illisible, peut-être : «quand »] il y a quelque chose de neuf, auquel il convient de s'opposer. Dans un an ou deux (s'il n'y a pas de guerre) ce sera mieux chez nous, sauf pour la pénurie de logement. Mais il sera plus difficile pour elle de devenir entièrement une des nôtres, intimement proche. Nous serons beaucoup plus indépendants sur les plans culturel et économique et, par conséquent, on n'aura plus tellement besoin d'aide.

Pourtant, malgré l'optimisme relatif aux perspectives de croissance économique, l'espoir d'un «avenir radieux » pour l'Union soviétique réside largement dans l'attente de la révolution mondiale provoquée par la «lutte des classes chez l'adversaire ». Aussi Spunde et Kravčenko suivent-ils de près le développement de la crise économique mondiale et la montée du fascisme, événements qui suscitent aussi bien l'appréhension de l' « encerclement impérialiste » et d'une guerre contre l'URSS que l'espoir d'un effondrement du système capitaliste.

De la retraite de Spunde (en 1932) à la guerre, la correspondance se fait considérablement moins soutenue. Les lettres conservées témoignent d'un certain repli sur des questions pratiques (organisation de la vie quotidienne, éducation des enfants) ${ }^{24}$.

24. L'éducation des enfants est cependant loin d'être une question anodine. Comme le montre la correspondance et les conversations de Spunde avec Jakov, ce rapport intergénérationnel a une dimension éminemment politique. 
Les correspondants se contentent souvent d'allusions pour en discuter " de vive voix ». Ils évitent apparemment certains sujets politiques. Néanmoins, les grandes questions qui concernent le développement du pays tout entier restent au premier plan de leurs préoccupations. Quand Kravčenko est en mission ou Spunde dans une maison de repos sur prescription médicale, on mesure bien dans leurs lettres à quel point ils ont du mal, surtout Spunde, à patienter jusqu'à la prochaine occasion de se parler directement et « faire part de [leurs] impressions ».

Les descendants de Spunde supposent qu'il aurait excipé d'une crise d'épilepsie lors d'une conférence de cheminots à Tokyo au début de l'année 1932, où il était le délégué soviétique pour le NKPS, pour justifier sa demande de mise à la retraite et éviter la collaboration avec un régime qu'il n'approuvait plus ${ }^{25}$. Tumaj Vite, un ami très proche de la famille depuis les années 1920 et à peu près du même âge que Jakov, affirme que Spunde «a haï Stalin pendant toute sa vie ». Il témoigne même d'un conflit ouvert avec Kaganovič, supérieur immédiat de Spunde et chef du NKPS, qui aurait exigé qu'il suive sans réserve toutes les instructions de Koba (Stalin). Alors, Spunde,

qui n'employait jamais de gros mots, l'a envoyé au diable. Il lui a expliqué qu'il n'agirait que sur les instructions de son jugement, de sa propre conscience et pour le bien de notre développement commun, mais jamais sur les instructions personnelles de quelqu'un. ${ }^{26}$

Néanmoins, même si ces récits sont authentiques et même si Spunde avait déjà à cette époque-là un avis aussi net contre le Secrétaire général, il n'était certainement pas (encore) un adversaire résolu du régime ou de la ligne du parti.

En 1936, Jakov quitte Moscou pour faire des études à l'institut d'aviation de Ejsk dans la région d'Azov. La correspondance familiale connaît une nouvelle phase : celle d'un échange épistolaire des deux parents avec leur fils ${ }^{27}$. La loyauté et le soutien que Spunde et Kravčenko apportent au système, indépendamment de ce qu'ils peuvent avoir pensé de la personne du Secrétaire général, s'y expriment. Dans ce contexte la correspondance Jakov-Spunde est particulièrement intéressante, dans la mesure où ils commencent (probablement en 1936 ou 1937) à mener régulièrement des «entretiens sur des thèmes sociétaux » (besedy na obščestvennye temy). Cet échange devient presque vital pour les deux interlocuteurs (selon leurs propres témoignages) et il se poursuit visiblement (d'une manière ou d'une autre) par écrit quand ils sont séparés. L'importance que revêt cet échange rend d'autant plus regrettable la disparition quasi totale des lettres de Jakov de cette période. Réponses et réactions de

25. Entretien avec Irina A. Korol'kova, deuxième femme de Jakov Spunde et avec Galina A. Spunde, petite-fille de Spunde (son père était né d'un premier lit), à Moscou (13 novembre 2004).

26. Tumaj Vite, «Pamjat’ », Neva, n 11, 2003, p. 186-201, ici p. 188

27. La majorité de ces lettres des parents au fils n'ont pas été déposées aux archives et sont restées dans la famille. Nous remercions Irina Korol'kova de nous avoir permis de les consulter. 
Spunde permettent néanmoins de voir combien Spunde, encore en 1936 et même en 1937, se situe comme acteur à l'intérieur du système. Voici quelques lignes de ce qu'il pense des perspectives d'une victoire du communisme dans un seul pays :

Maintenant à propos de ta question sur le communisme [...] La construction du communisme dans notre pays est tout à fait possible, même au cas (particulièrement peu probable) où il nous faut parcourir seuls tout le chemin de la première phase jusqu'à la pleine victoire, encore avant l'avènement d'une révolution prolétarienne dans une série de pays, sinon dans le monde entier. On a tout ce qui est nécessaire pour ça, une bonne base industrielle, et maintenant une agriculture socialisée. Il nous reste deux classes, il est vrai, la classe ouvrière et la paysannerie, mais ce sont des classes amicales, surtout maintenant, où l'une comme l'autre vit sur la base d'une économie socialisée. Maintenant il est évident pour tout le monde, qu'à l'intérieur de l'URSS, toute base sociale (obščestvennaja baza) pour une contre-révolution a disparu. Il ne reste que la possibilité d'actions hostiles de la part d'individus isolés, ou bien de tous petits groupuscules. Mais décidément (rešitel'no) sans le moindre appui et sans la moindre possibilité d'appui dans la masse populaire. (25.12.1936)

«Agriculture socialisée », classe ouvrière et paysannerie comme «classes amicales », «toute base sociale pour une contre-révolution a disparu », il faut se rappeler que Spunde écrit cela sur fond de répressions de masse qui prennent de plus en plus d'ampleur : les koulaks revenus de leur exil sont de nouveau persécutés et le deuxième procès de Moscou contre Radek, Pjatakov, etc., se prépare. Le premier, contre Kamenev, Zinov'ev et d'autres, a également été discuté par Spunde et son fils. En réponse à une question de Jakov, Spunde lui donne des renseignements biographiques sur les inculpés, sur leurs adhésions à l'opposition trotskiste. Il évoque ses souvenirs. Mračkovskij se serait, déjà en 1922, montré indifférent au développement (extrêmement inquiétant selon Spunde) d'une discipline de plus en plus aveugle au sein de l'Armée rouge et aurait affirmé qu'il ne s'intéressait pas à la politique, qu'il était « seulement » militaire, sur quoi Spunde aurait pratiquement rompu le contact.

Bref, il avait déjà oublié qu'il est important d'avoir une connaissance exemplaire de l'art militaire, mais que ce n'est qu'un moyen dans la lutte pour le communisme. Autrement on glisse vers une position de classe hostile (naše spolzanie na poziciju klassovo-vraždebnuju). (10.09.1936)

En dépit de l'emploi de mots clés comme «contre-révolution », «lutte contre le parti » « union avec le fascisme » (voir ci-après), etc., le ton de la lettre reste plutôt sobre et modéré (eu égard aux épithètes qu'on a généralement données aux inculpés et à l'énorme retentissement du procès). Comme s'il avait rempli une obligation, Spunde conclut sa lettre en encourageant son fils à former son propre jugement à partir des sténogrammes des différents congrès du parti tenus quand les accusés étaient encore des acteurs importants de la vie politique du pays :

J'ai répondu à tes questions. Les accusés du procès étaient tous depuis longtemps des partisans de Trockij - Zinov'ev - Kamenev. Tous sont des gens qui 
ont commencé leur lutte contre le parti depuis longtemps et qui ont ensuite glissé vers une contre-révolution de plus en plus ouverte, jusqu'à une union avec les fascistes. Mais il vaut mieux t'en convaincre en prenant connaissance de l'histoire du parti. Dans la bibliothèque, chez vous, il y a très probablement les procès-verbaux des congrès du parti. Là-bas tu retrouveras beaucoup de ces noms. Pour cela je te renvoie à la source originale. (10.09.36)

Mais revenons à la lettre de Spunde du 25 décembre 1936 où il traite des perspectives de la construction du communisme en Union soviétique :

S'agissant du passage d'une société construite sur le principe «chacun donne selon ses capacités, chacun reçoit selon son travail »à une société qui vivra d'après le principe « chacun donne selon ses capacités, chacun reçoit selon ses besoins », il faut seulement arriver à une croissance des forces productrices et à une croissance de la richesse nationale, mais à une croissance gigantesque, pour laquelle il faut même avec notre vitesse de développement une échéance considérable (porjadočnyj srok). Nous sommes déjà riches, en comparaison de 1932 par exemple ${ }^{28}$, mais même maintenant nous sommes encore pauvres en comparaison de ce qu'exige le communisme. Il nous faut également une rééducation très, très profonde et plus étendue de notre peuple, car même maintenant les vestiges du capitalisme dans notre conscience sont encore nombreux. Mais chez nous il y a tout ce qui est nécessaire. C'est dire qu'il s'agit seulement des échéances. Et les échéances dépendent maintenant de plus en plus de nousmêmes, de notre persévérance, de notre capacité à bien travailler.

À en juger par ta lettre c'est la question de l'armée qui vous ${ }^{29}$ trouble. Quelques mots là-dessus. La présence de l'armée est le résultat de notre coexistence avec le monde capitaliste. Mais même maintenant, alors que nous avons inscrit dans notre constitution que nous vivons d'ores et déjà le socialisme, que celui-ci est déjà solidement installé (kogda my v našej konstitucii zafiksirovali togo, čto socializm u nas uže nalico, uže ustanovilsja), tout cela se passe alors que sur les six septièmes du globe terrestre règne toujours l'ordre capitaliste, et dans d'autres parties du monde (comme en Abyssinie, p.ex.) même un ordre précapitaliste. C'est-à-dire que nos relations mutuelles avec le monde extérieur ne peuvent pas être conséquemment socialistes. Aussi dans les relations multilatérales nous partons des principes socialistes, mais il faut hurler avec les loups. Et voilà la présence de telles contradictions dans les relations avec le monde extérieur ne change aucunement la base socialiste et la nature socialiste de notre ordre. On peut dire la même chose au sujet du capitalisme, s'il survit jusqu'au moment de la transition de l'URSS au communisme.

28. En renvoyant à 1932, Spunde fait référence à la veille de la grande famine (1933-1934) qui a coûté des millions de vies humaines, et cela à cause de la collectivisation forcée où l'on ne s'est pas contenté de confisquer aux paysans leurs récoltes, mais également les réserves de semences.

29. Nous attirons l'attention sur le pluriel, qui atteste que Spunde est loin d'être le seul interlocuteur de son fils, aussi en ce qui concerne ces questions qui étaient sur le point d'être démodées, surtout après la ratification de la constitution stalinienne et la fin de la grande discussion qu'elle a suscitée. Jakov semble également avoir largement discuté les problèmes de l'avenir communiste dans le cercle de ses amis et camarades. 
Cela illustre à quel point les correspondants tiennent fermement à leurs idéaux et, comme Spunde l'appellera plus tard, aux «promesses » de la révolution, c'est-àdire au projet d'édification d'une nouvelle société communiste sur la base des acquis de l'Union soviétique des années 1930. Même s'il s'agit ici apparemment de lettres envoyées par la poste, nous avons tendance à ne pas taxer ces réflexions de duplicité. Certes, des sujets importants sont visiblement omis, mais il faut prendre au sérieux tout ce que les interlocuteurs disent. S'ils s'étaient écrit uniquement pour démontrer leur loyauté au régime, ils n'auraient pas laissé place à des ambiguïtés, voire à des expressions de mécontentement, comme ici sur la politique d'information sur la guerre d'Espagne, qui est un des grands thèmes de la correspondance :

[...] Je suis en colère contre nos journaux. L'information est mal fournie. L'agence Tass et la Pravda ont toutes les deux leurs envoyés spéciaux là-bas [en Espagne]. Mais jusqu'ici il n'y a aucune information précise et claire sur le programme sociopolitique du front populaire, sur les rapports entre les partis à l'intérieur du front uni. Nous ne savons pas, si on a au moins déjà donné aux paysans la terre des propriétaires fonciers qui sont morts dans les combats. Nous ne savons pas comment la question nationale sera résolue au Maroc, etc. Pourtant, dans ces questions se trouve la clef du succès politique. (26.09.36)

Bien que sa critique vise juste (notamment en ce qui concerne les silences de la presse soviétique sur la guerre d'Espagne), ce sont là «grognements » d'un partisan profondément engagé, décidé à promouvoir la cause qu'il voit remise en question par une négligence inadmissible.

La correspondance montre bien comment les interlocuteurs - par le fait même de la communication engagée, voire la controverse - se confortent dans leur soutien au système et dans leur dévouement à la cause du grand avenir, de la création d'une nouvelle société. Le seul fait d'échanger des points de vue vaut déjà un engagement et oriente les interlocuteurs vers l'action, ce qui confirme, par conséquent, leurs opinions et jugements (ce n'est pas le cas dans la pratique solitaire du journal).

\section{Un positionnement critique à partir de l'échange dans le cercle familial}

Le problème de l'activité, de la praxis - élevée au premier rang dans la conception marxiste, contre la pure spéculation de l'idéalisme - est directement abordé dans une lettre de 1940 ou $1941^{30}$, qui fait transparaître déjà un rapport au système bien plus complexe. L'attitude de Spunde a-t-elle changé entre-temps ?31. Ce rapport

30. La lettre est sans date ni lieu, mais Spunde mentionne l'âge de Jakov au moment de la rédaction (il s'agit de « la 23e année » de sa vie). On peut déduire que la lettre est écrite entre août 1940 et août 1941. Spunde écrit que quatre ans sont passés depuis qu'ils ont commencé leurs entretiens politiques en 1936 ou 1937.

31. Depuis mars 1938, une perspective plus « extérieure » lui est quasiment imposée par le fait de son exclusion du parti. Et que dire des bouleversements qui se sont produits en URSS et dans le monde entier et qui ont évidemment laissé des traces profondes chez des observateurs et acteurs comme lui ? 
plus complexe au système n'a-t-il pu être exprimé dans les courriers postaux précédents? Non, depuis 1938 Jakov habite de nouveau chez ses parents à Moscou - où il est admis à l'Institut d'aviation (MAI) en septembre 1940 - et sans aucun doute la lettre a été transmise personnellement. Il s'agit d'un des rares cas où Spunde préfère la forme écrite à l'orale; d'ordinaire, il ne s'y résout que forcé par l'éloignement de ses interlocuteurs. Spunde prend ici du recul par rapport au contenu de leurs entretiens « sur des thèmes sociétaux » afin d'en problématiser les enjeux et les objectifs (qualifiés un peu étroitement de «pédagogiques »), à savoir l'élaboration d'une réflexion indépendante et d'une conception du monde réfléchie chez son fils, ou bien, pour le dire avec ses mots, vyrabotka vzgljada na vešči, ou même vyrabotka gluboko produmannogo $i$ vystradannogo mirovozzrenija. En raison de ce changement de perspective, les opinions sur les événements et sur le caractère du régime stalinien, qui sont à l'évidence traités largement dans leurs échanges oraux, n'apparaissent ici qu'entre les lignes. Inquiet de « la passivité » de son fils, il le raisonne :

Si tu ne trouves pas en toi la force d'être actif malgré une vie pesante (vopreki davjaščej žizni), alors c'est un moindre mal (je répète il s'agit d'un mal, mais d'un moindre mal) de rester un outil aveugle du processus objectif, puisque dans ce cas-là tu seras au moins un être actif, bien qu'inconscient. Car il n'y a rien de pire qu'un intellect qui réfléchit et comprend, mais qui est complètement incapable d'agir (une sorte de Bernard Shaw, mais à une bien moindre échelle ${ }^{32}$ ).

Souvent ton comportement me fait souffrir jusqu'à la douleur à cause de ce dilemme. Tu tombes souvent dans un état de passivité, qui fait transparaître ee [rayé dans le manuscrit] cette même médiocrité et ce même égoïsme, qui est tellement caractéristique de la vie actuelle, seulement dans la variante de celui qui «comprend». Je pensais réveiller en toi la volonté passionnée et invincible de savoir comment vivre et agir malgré tout. Mais assez souvent c'est le contraire qui arrive. Dans aucun domaine je ne ressens (chez toi) un penchant suffisamment fort pour briser les barrières. [...]

Suivent des remarques critiques concrètes sur le comportement de Jakov: son manque de sérieux dans la lecture de Marx, son manque de motivation pour des études qu'il a cependant lui-même choisies, et sur sa paresse et sa négligence à la maison. Bref, des traits d'un oblomovisme qui sont loin d'épuiser toute la complexité du dilemme que Spunde a brossé auparavant en termes relativement abstraits, à savoir le problème de (re)trouver une praxis appropriée à la compréhension des (nouvelles, depuis quand ?) contradictions du système environnant. Après avoir menacé Jakov de cesser leurs entretiens politiques et de le laisser ainsi libre de tomber dans « le moindre mal » de «l'outil aveugle », s'il continuait à «s'accommoder du rôle de

32. Que Spunde veut-il dire par cette comparaison ? C'était apparemment un sujet dont ils avaient déjà débattu. On sait que George Bernard Shaw fut extrêmement actif et productif jusqu'à la fin de sa vie (il décède en 1950 à 94 ans). Spunde ferait-il allusion à l'attitude laudative de Shaw à l'égard du régime stalinien ? Spunde est-il déjà parvenu au point d'exiger d'un « révolutionnaire conséquent» (étranger qui plus est) la praxis du résistant contre le régime qu'il n'élaborera que dans une lettre de 1941 à sa femme, que évoquée plus loin ? 
petit-bourgeois qui comprend [quand même] quelque chose » durant «la période petite-bourgeoise de sa vie» ( $s$ rol'ju «koe-čto ponimajuščego » meščanina $V$ meščanskuju polosu žizni) - , il introduit un motif, qui sera une des pierres angulaires de la critique qu'il élaborera bientôt par écrit et de façon plus nette, à savoir son indignation face au Précis d'histoire du parti (Kratkij kurs istorii partii).

C'est surtout à partir de la guerre que des orientations dissidentes sont clairement exprimées dans les lettres. Plus les interlocuteurs prennent leurs distances par rapport au système stalinien, plus ils font référence à l'époque révolutionnaire, même si la révolution et sa mémoire ont déjà joué un rôle clé dans leur correspondance antérieure. Or, ils passent à présent de l'échange communicationnel à une élaboration écrite dans le cadre d'un énorme effort d'écriture mémorielle. Cette écriture n'interrompt cependant pas la communication familiale à laquelle Jakov prend une part active. Curieusement, c'est aussi pendant la guerre que l'on voit naître chez beaucoup d'autres vieux bolcheviks survivants une activité similaire d'écriture mémorielle. Elle se distingue par le même accent chronologique sur l'époque révolutionnaire. Spunde et Kravčenko ne font donc pas exception ${ }^{33}$. Ce qui rend leur cas exceptionnel, c'est non seulement la rigueur des jugements, de Spunde surtout, mais aussi (et en premier lieu) le fait que l'on peut retrouver la genèse de cette écriture dans l'échange engagé dans le cercle familial.

Peu après l'invasion allemande, Spunde et Kravčenko sont évacués à Molotovsk dans la région de Kirov (Vjatka), où ils habiteront ensemble jusqu'en mai 1942, date à laquelle Kravčenko est mutée à Kirov puis, en 1943, à Moscou pour travailler à la Bibliothèque Lenin. Spunde reste presque jusqu'à la fin de la guerre à Molotovsk où il travaille comme comptable en chef de la succursale du Sel'elektro. Jakov est évacué avec le MAI à Alma-Ata où il continue sa formation jusqu'à l'été 1942 date de son incorporation dans l'armée ${ }^{34}$. Au début de la guerre, l'échange direct et, par conséquent, le plus intensif, a donc lieu entre les deux époux à Molotovsk. La communication avec Jakov, moins soutenue à cause de l'éloignement, a logiquement laissé des traces écrites beaucoup plus importantes (jusqu'au départ de Jakov sur le front en août 1942), mais apparemment avec un éventail réduit car elle emprunte la voie postale. Le thème du cours de la guerre prédomine et, même si la préoccupation de la défense du pays contre l'envahisseur n'est pas du tout feinte, nous avons ici, à la différence des années 1930, des preuves d'autocensure (au sens

33. Pour les manuscrits de Kravčenko, cf. GARF, f. R-9665, op. 1, d. 55, 58, 60-61, 63, ainsi que ses souvenirs nombreux sur Krupskaja: ibid. d. 52-54 et Dom Plehanova (Rukopisnyj otdel Publičnoj biblioteki St-Peterburga - Département des manuscrits de la bibliothèque publique de Saint-Pétersbourg), f. 1119, op. 1, d. 84-107. Pour les manuscrits de Spunde cf. GARF, f. R9665, op. 1, d. 201-208. À en juger par les renvois de Jakov, il a dû y avoir encore d'autres manuscrits qui ne sont plus conservés ou bien sont déposés dans d'autres archives privées, étant donné que ses manuscrits ont circulé, ne serait-ce que dans des cercles limités. Ainsi V. Barančenko (Gaven, M. : Molodaja Gvardija, 1967) et Roy Medwedew (Let History Judge : The origins and consequences of Stalinism, New York : Columbia University Press, 1989, vol. 1) ont consulté au moins des parties des Mémoires de Spunde, bien avant que le matériau n'ait été déposé aux archives d'État par Irina Korol’kova, la veuve de Jakov (entre 1991 et 1994).

34. Cf. son autobiographie, GARF, f. R-9665, op. 1, d. 274, 1.3 ; ainsi que sa lettre à Kravčenko du 27.08.1942, ibid, d. 268,1. 28. 
d'omissions). Nous pouvons en effet comparer ces lettres envoyées en Asie centrale avec un nouveau « message » de Spunde (il parle de poslanie), cette fois-ci à sa femme, qu'il lui a remis en mains propres à Molotovsk (28.12.1941). Il y avait apparemment entre eux un différend politique sérieux qui l'a incité à mettre sur le papier ses arguments dans une sorte de petit mémorandum ${ }^{35}$. Il y expose les problèmes qui le hantent et qu'il ne peut pas évoquer dans sa correspondance avec Jakov : les contradictions profondes de l'époque, la rupture du régime soviétique avec la légitimité révolutionnaire et la question troublante et foncièrement morale de savoir comment peut-on — ou plutôt comment faut-il - agir maintenant en tant que vrai révolutionnaire. Il pose donc la fameuse question : «que faire ? déjà évoquée dans son mot (zapiska) à Jakov à propos du Précis d'histoire.

Jakov, de son côté, n'oublie apparemment pas une promesse faite au sujet de ce qui lui tient particulièrement à cœur. Dans une carte postale du 20 février 1942 (qui, comme la plupart des lettres de Jakov, n'est pas conservée, mais que Kravčenko a presque intégralement recopiée dans son journal ${ }^{36}$ ), il expose son point de vue avec beaucoup de conviction. En renvoyant à sa propre génération, il esquisse ce qu'il tient pour les obligations morales de la génération de ses parents :

[...] Je vais te raconter un tas de choses sur ce que pensent maintenant dans mon entourage les jeunes (rebjata), quel est leur rapport à la vie. Je te prie instamment de consacrer tout le reste de tes forces (et il t'en reste peu) à l'écriture des livres dont je t'ai parlé. Tu as promis de faire cela [...] au nom de tous ceux qui ne sont plus en état de le faire.

Mère et fils échangeaient donc aussi des promesses. Dans ces livres à écrire, Kravčenko doit parler de la révolution. Ceux au nom desquels elle a promis d'écrire sont ses camarades révolutionnaires morts, au moins en partie éliminés par les purges, comme on doit bien le supposer.

Chaque jour, je pense avec peine à la manière dont tes forces et celles de papa sont gaspillées en vain, et cela dans un moment où le désir indomptable et irrésistible de trouver ces livres peut apparaître aujourd'hui, demain (peut-être dans une semaine), et [...] susciter du mépris envers ceux qui ne les ont pas écrits (i notki prezrenija k tem, kto ne napisal ih).

35. Cette «lettre » a été publiée isolément : A.P. Spunde, «"Najti smelyj, čestnyj otvet..." Zlobodnevnoe pis'mo poluvekovoj davnosti » ["trouver une réponse audacieuse, honnête..." Lettre de cinquante ans d'âge à l'ordre du jour], Kommunist, n 7, 1990, p. 5-7. L'original, à la différence de la zapiska à Jakov, est daté (28.12), mais sans indication d'année. Les éditeurs l'ayant datée de 1943 (ce qui est erroné), suggèrent qu'elle aurait été envoyée par la poste à Moscou, où Kravčenko travaillait en 1943 à la Bibliothèque Lenin. Dans l'historiographie, cette publication a été jusqu'ici totalement ignorée (sauf par Vadim Z. Rogovin, Stalinskij Neonep [La néo-Nep stalinienne], M., 1994, p. 239-241) et à notre avis pour deux raisons : d'une part, on avait implicitement tendance à tenir pour fou quelqu'un qui envoyait de tels documents par la poste ; d'autre part, ce qui n'est pas contradictoire, on y a vu un phénomène singulier : le résultat d'une réflexion libre d'un individu autonome extrêmement lucide et par là exceptionnel.

36. Cf. GARF, f. R-9665, op.1, d.43,1.32. 
La formulation est sans équivoque sur l'impact de l'échange avec Spunde dont n'est cependant pas exclue l'exhortation contre le «gaspillage » d'énergie «en vain ». Par la suite, Jakov renvoie à ses entretiens personnels avec ses camarades à l'Institut d'aviation :

Et voilà dans mes entretiens avec les gens j'entends très souvent : « il faudrait lire un livre intéressant, pas creux, sur la façon dont tout était (pročest' interesnuju i ne pustuju knigu o tom, kak vse bylo). Et cela de la part de ceux qui ont d'énormes a priori sur toutes les questions sociétales. (20.02.1942)

On voit dans quelle atmosphère de franchise et d'ouverture Jakov et ses camarades se rencontrent et que même des points de vue très différents sur les «questions sociétales » ne les empêchent pas de débattre de sujets aussi délicats que la représentation officielle de l'histoire, notamment celle de la révolution à laquelle Jakov se réfère ici.

Beaucoup plus qu'au projet de popularisation de l'Anti-Dühring de Friedrich Engels, qui tenait à cœur à sa mère, Jakov s'intéresse à son travail sur la biographie et la réédition des œuvres complètes de Krupskaja qui avaient déjà été, de son vivant, censurées sans merci : Kravčenko a retrouvé dans les originaux le crayon bleu du censeur ${ }^{37}$ ! Une lettre de Jakov à sa mère d'Alma-Ata non datée (c'est-àdire de l'évacuation en 1941 ou 1942) précise ce sujet :

Je pense quand même, que ton travail sur N.K. [Krupskaja] est très important dans tous les cas et voici pourquoi. Seulement toi (ou quelqu'un de ta génération) peut écrire sur elle, écris non seulement sur toute la somme d'idées qu'elle a propagées (et toi aussi d'ailleurs), mais également sur toute la somme de rêves ( vsju tu summu mečtanij) (parfois assez prématurés, comme la vie l'a montré), qui se sont reflétés dans ces idées. Ces derniers [les rêves] peuvent se transformer facilement en un cadavre insignifiant (stat' neznačimym trupom) ${ }^{38}$.

Jakov s'inquiète des rêves et des utopies révolutionnaires qui risquent d'être détruits et que seule la mémoire des participants aux événements peut rétablir. D'où l'insistance avec laquelle il exhorte ses parents à écrire leurs Mémoires, que ce soit sous forme de souvenirs sur Krupskaja ou bien plus directement sur leur propre vécu. Il est évident que dans le cadre de la famille ils en ont déjà beaucoup parlé. Ses propres discussions avec ses parents ont convaincu Jakov qu'ils ont bien autre chose à apporter à la mémoire de la révolution que les ouvrages de la commémoration officielle (qu'il appelle «livres creux »). Qu'ont-ils donc à dire ? Selon le « message » du 28 décembre 1941 de Spunde à sa femme, il s'agit de trouver des

37. Cf. ce qu'elle note dans son journal le 23.9.1941 (GARF, f. R-9665, op. 1, d. 23, 1. 39). On peut supposer que Jakov est au courant de ces découvertes, puisqu'ils se sont vus le 5 novembre 1941 pour la dernière fois avant l'incorporation de Jakov dans l'armée. Cf. l'entrée du 11.5.1941 dans le même journal.

38. Pour les quelques lettres conservées de Jakov, voir GARF, f. R-9665, op. 1, d. 268. Le « cadavre» (trup) rappelle aussi le choix des mots de Spunde, qu'on verra encore dans son « message » à Kravčenko dans une comparaison de l'Anti-Dühring avec l'Église. 
réponses aux questions pressantes du temps présent. Sous forme d'interrogations, Spunde dresse le catalogue des écrasants jugements sur la vie présente :

Pourquoi les bolcheviks, combattants et organisateurs qui ont donné aux gens l'énorme étendue de l'État bolchevik, pourquoi ces bolcheviks (il est vrai qu'ils n'en portaient alors que le nom) ont-ils détruit les soviets [...], et sont-ils ainsi descendus plus bas que les meilleurs États bourgeois dans le domaine de la politique générale, mais ont abusivement gardé le nom des Soviets ?

Pourquoi est-on arrivé à la contradiction vitale suivante ? Le socialisme a été pensé par Marx, Engels, Lenin et par tout le collectif bolchevik comme le grand épanouissement de l'activité indépendante et de l'initiative humaines. Et l'accomplissement du processus de nationalisation de l'économie était censé éliminer les entraves de toutes les forces de classes égoïstes. - Et puis, dans la vie, en réalité, on est arrivé au contraire. Quand nous avons collectivisé seulement l'industrie, cela a [vraiment] libéré (razvjazalo) l'initiative des masses, mais quand nous avons en plus créé une agriculture collectivisée, l'oppression de l'appareil étatique a atteint des limites connues seulement pendant les périodes les plus réactionnaires du féodalisme et sous le règne du capitalisme uniquement durant des périodes historiques de courte durée.

Pourquoi les bolcheviks, qui ont très bien compris qu'on ne peut donner de la vraie joie et un vrai élan au peuple que si l'on emprunte la voie de la réalisation des revendications qui émergent indépendamment d'en bas [n'ont-ils pas emprunté cette voie ?]. Tout type de voie «bismarckienne» (Ivan le Terrible, Pierre le Grand, Bismarck, etc.) remplace le vieux joug par un nouveau, certes qui est progressiste, mais il n'en est pas plus léger : pourquoi les bolcheviks sont-ils devenus les chantres de Pierre, Ivan le Terrible, Bismarck, etc. ?

Pourquoi les bolcheviks, qui ont honnêtement mené une politique de paix jusqu'en 1934 environ, se sont-ils faits complices (součastniki) de l'embrasement (razžiganie) de la guerre et prennent-ils part directement au commerce de peuples entiers comme du bétail sur un marché à viande ?

Cette irruption d'amertume ne manque pas de clarté : c'est une condamnation fondamentale du système stalinien, qui est loin de se contenter d'une dénonciation d'un pur «culte de la personnalité »: Spunde ne mentionne jamais le nom de Stalin, ses questions concernent la genèse du stalinisme comme système, ou de ce qu'il appelle «une voie bismarckienne » ${ }^{39}$ qui a rompu avec les idéaux et les «promesses de la révolution ». Il attribue la responsabilité de cette dégénérescence aux «bolcheviks », tout en reconnaissant clairement sa propre complicité à l'époque, notamment quand il parle de la collectivisation. Il bascule alors d' « eux » (oni) à « nous » (my) inclusif (« quand nous avons collectivisé... »).

Mais comment réagir? On se souvient comment il a rebattu les oreilles à Jakov quand il pensait que celui-ci n'avait pas tiré les conséquences pratiques de son intel-

39. Il fait apparemment référence à des publications toutes récentes et bien disposées à l'égard de Bismarck qui sont plus ou moins le fruit du rapprochement avec l'Allemagne nazie à la suite du pacte de non-agression. On a publié ainsi ses Mémoires dans leur intégralité avec une préface de A.S. Erusalimskij : Otto Bismark, Mysli i vospominanija [Otto Bismarck, Pensées et Mémoires], 3 t., M. : OGIZ Gosudarstvennoe social’no-ekonomičeskoe izdatel'stvo, 19401941, ainsi qu'une biographie politique de V.G. Revunenkov, Prihod Bismarka $k$ vlasti [L'arrivée de Bismarck au pouvoir], L., 1941. 
ligence. «Que faire » donc à présent ? Recourant à une comparaison avec la réaction sous Stolypin, Spunde ne voit pas d'autre issue que le travail clandestin.

Les marxistes ont déjà compris à l'époque de Stolypin (vremena stolypinščiny) que si ce n'est pas la voie révolutionnaire (au sens d'une révolution profondément populaire), mais la voie bismarckienne qui est suivie, alors il est inévitable et absolument nécessaire pour des révolutionnaires conséquents avec euxmêmes de passer à un travail «noir ». Dans ce cas-là c'est la seule chance de trouver une issue. [...] Mais que cela veut-il dire concrètement ?

Puisqu'il considère que « les complots et tentatives héroïques d'un petit nombre de gens visant à raccourcir le cours de l'histoire par une convulsion artificielle sont inéluctablement voués à l'échec », il propose avant tout un projet d'instruction où il s'agirait de dire la vérité « aux masses », un peu comme « les milliers d'intellectuels (intelligenty) » qui «sont partis pour un travail sur le terrain (na zemskuju rabotu) » après 1881. Concrètement, pour le présent :

Dans tous les domaines, nous avons extrêmement besoin d'une analyse authentique, consciencieuse de l'histoire de notre travail ${ }^{40}$. Elle est nécessaire aussi pour les masses populaires. Dans un tel travail la seule présentation des faits peut montrer énormément de choses, on peut relever toutes les questions que la vie a posées.

Il s'agirait donc de vérifier et revérifier scrupuleusement tous les faits dans le cadre d'une nouvelle histoire totale de l'histoire soviétique depuis la révolution, sans formules toutes faites, «ne seraient-ce que des recettes marxiennes ou léniniennes ». Il y inclut une proposition concrète à sa femme : « Je t'ai déjà parlé d'une recherche sur l'histoire de l'école soviétique. J'y reviens encore une fois. » Kravčenko devrait travailler dans les archives du Narkompros pour écrire une histoire de l'instruction publique en Union soviétique, ou plutôt celle de son déclin.

Et si tu avais suffisamment de force pour embrasser les deux décennies de 19171927 et de 1927-193741, on arriverait à un contraste extrême. La grandeur de la première époque se révélerait tout à fait clairement.

40. Ses Mémoires montrent qu'il n'a pas abandonné ce projet par la suite. Il revendique une vérification profonde des faits et on remarque qu'il met sur le même plan étude historique critique («objective ») et reconsidération « subjective », ce que sont les Mémoires personnels. «Il faut un travail fondamental, objectif et subjectif, pour que l'on puisse avoir pleine connaissance de la sortie sur une voie progressive (čtoby byl osoznan vyhod na postupatel'nom puti). Avec ces écrits [qui sont des Mémoires], je veux y contribuer de toutes mes forces ». GARF, f. R-9665, op. 1, d. 203,1. 19-21.

41. Dans ses Mémoires, Spunde fait de l'année 1927 une ligne de démarcation, quand il brosse une périodisation de 1'histoire soviétique. Il y distingue la lutte clandestine contre le tsarisme, la révolution et la guerre civile, les années de la construction pacifique 1921-1927 (c'est-à-dire la NEP), puis la reconstruction (perestrojka) qui, malgré la progressivité « objective » du développement économique, n'aurait déjà plus été l'affaire du peuple (uže ne narodnaja) mais le règne de l'appareil d'État qui ferait du peuple le rouage d'une machine (GARF, f. R-9665, op. 1, d. 203, 1. 19-21), ce qui correspond tout à fait aux fameux vintiki de Stalin qui les présente cependant comme un grand acquis. 
Il conclut sa lettre en renvoyant à l'exemple de Jakov pour justifier sa foi dans le pouvoir de la persuasion :

Quand j'ai cité à Jaša, pendant notre travail ensemble à Moscou, les faits concrets de notre économie et de notre quotidien (et je n'ai pas parlé de mémoire, mais cité exactement les documents), cela a fait sur lui l'effet d'une bombe.

Et, véritablement, Jakov n'a jamais oublié ces entretiens. Comme il l'écrira plus tard : «Comme mon père l'avait compris avec beaucoup d'acuité, les plans quinquennaux de Stalin n'ont jamais été réalisés. ${ }^{42}$ La métaphore de la bombe ne laisse aucun doute sur le message implicite : pour «tenir nos promesses » d'Octobre ${ }^{43}$, il faut renverser le régime stalinien.

Spunde ne s'en tient pas à harceler sa femme et son fils. Jusqu'à la fin de sa vie, il va travailler frénétiquement tant sur l'histoire russe et soviétique que sur ses propres Mémoires. Son objectif est toujours de contester la représentation officielle de l'histoire et de transmettre à la jeune génération (en qui il met tout son espoir) l'esprit et l'expérience de la révolution tels que lui et les autres membres de sa génération les ont vécus. D'où aussi le poids qu'il accorde au jugement de son fils, représentant de cette jeune génération qu'il ne considère pas que comme le simple destinataire des vérités que les représentants de sa génération ont à lui léguer. Il recherche constamment l'échange avec elle, veut apprendre d'elle. Ainsi dans une lettre à Jakov de 1950, il envoie un brouillon de ses Mémoires et exprime son inquiétude :

si le récit de ma vie a vraiment un intérêt, s'il peut intéresser quelqu'un, s'il suscite ou renforce l'intérêt pour ce par quoi et comment ma génération a vécu [interes $k$ tomu, čem i kak žila moe pokolenie], où sont les régularités de notre essor impétueux et de notre chute [gde zakonomernosti našego burnogo vzleta $i$ našego padenija].

Il affirme avoir suivi le conseil de son fils en choisissant la trame chronologique et autobiographique au lieu de partir de jugements généraux. Néanmoins, soucieux de la «représentativité » de ce qu'il écrit, il craint toujours l'inflation excessive du «moi» (legko sbit'sja na jakan'e), qui risquerait d'empêcher de lier le «particulier» au «général». La lettre, toute pleine d'excitation et d'angoisse, montre bien combien Spunde tient à l'opinion de son fils ${ }^{44}$.

42. Ainsi dans une lettre de 1967 aux éditeurs d'une revue (impossible de préciser laquelle) où Jakov propose une partie des Mémoires de son père pour publication et évoque l'attitude de Spunde vis-à-vis de Stalin (GARF, f. R-9665, op. 1, d. 276, 1. 4).

43. Cf. ses Mémoires : une petite partie est publiée après la mort de Spunde à l'occasion du $50^{\mathrm{e}}$ anniversaire de la révolution d'Octobre (à l'initiative de Jakov et de sa mère), Novyj Mir, 10 (1967), p. 184-198, ici p. 189.

44. Lettre du 5 avril 1950 (GARF, f. R-9665, op. 1, d. 225, 1. 28-29). 


\section{Enjeu et mise en valeur de l'expérience révolutionnaire?}

Quel est donc l'enjeu de cette écriture presque frénétique dont l'objet est en tout premier lieu l'expérience révolutionnaire ? Pourquoi les interlocuteurs accordentils une telle importance à la révolution ? Comment peut-on expliquer qu'il y ait tant de versions différentes (non pas contradictoires mais nombreuses), pourtant pratiquement toutes conçues comme narrations de la vie entière jusqu'au présent mais qui s'arrêtent aussi toutes aux années 1920, ou au plus tard en $1926^{45}$ ? Posons ces questions à deux niveaux différents. Condensons, en un premier temps, les propos des acteurs eux-mêmes, surtout ceux de Spunde, tels qu'ils apparaissent dans les nombreux manuscrits de ses Mémoires afin d'en mieux faire ressortir le principe qu'il met en avant contre le régime en place. Puis interrogeons-nous au regard de l'expérience révolutionnaire qui, bien que jamais explicite - tant le cadre cognitif de ses propres acteurs est impuissant à la conceptualiser — n'en renvoie pas moins à un conflit sous-jacent de l'interprétation de la révolution.

1. D'après les passages cités plus haut, la révolution se définit surtout pour Spunde et sa famille par les idéaux et les buts pour lesquels sa génération a lutté : la création d'une société sans classes, pleine d'initiatives indépendantes et spontanées, où les intérêts égoïstes disparaîtront et où les gens ne travailleront que de leur propre gré. Comme le souligne Spunde dans ses Mémoires, il ne lui importe pas tant qu'aucun de ces idéaux ne soit (encore) réalisé, que l'Union soviétique soit toujours bien en arrière par rapport aux pays occidentaux, en termes économiques par exemple. Ce qu'il dénonce en tout premier lieu, c'est l'arbitraire, le mensonge et le cynisme avec lequel les dirigeants du parti proclament la réalisation de ces promesses révolutionnaires en dépit de la réalité. Quand Spunde évoque la suppression du pouvoir des soviets, il affirme que garder le nom de «soviets » était une imposture (« on a abusivement gardé le nom de soviets »). Il démontre à Jakov que les bilans de tous les plans quinquennaux ont été falsifiés pour que le régime de Stalin puisse annoncer leur dépassement et leur réalisation en quatre ans. Et dans cette même veine, Spunde conclut son message de la fin de l'année 1941 à sa femme (juste après la remarque sur la bombe) sur ces mots : « À ce point notre histoire - toute récente pourtant - est encore calomniée par toutes sortes de Précis d'histoire (Nastol'ko obolgana stol' ešče, kazalos', svežaja istorija vsjakimi «Kratkimi kursami».) Spunde voit donc dans le stalinisme un système du mensonge, système qui brouille systématiquement les faits, qui a constamment recours à la falsification et interdit le recours à des faits inopportuns. Le Précis d'histoire en est le symbole et la pierre d'achoppement, notamment en ce qui concerne la mémoire de la révolution. Tout à fait consciemment Spunde écrit donc contre ce manuel d'histoire, contre cette représentation officielle devenue

45. GARF, f. R-9665, op. 1, d. 205, dans ce manuscrit, Spunde arrive à raconter son parcours jusqu'en 1926. Bien des digressions anticipent sur des événements tardifs, notamment lorsqu'il s'agit d'expliquer ce qu'est devenu tel ou tel révolutionnaire, avec qui il avait affaire pendant les années de la révolution. Dans la plupart des cas ils ont péri dans les répressions staliniennes. 
obligatoire et incontournable pour tous les étudiants, aussi bien dans les sciences historiques que dans les autres disciplines.

Par rapport au stalinisme, l'époque révolutionnaire (que la jeune génération n'a pas connue) est ainsi liée à la vie et l'agir dans un monde réel où l'on pouvait librement rappeler les faits dans les discussions politiques. Toutes les utopies et finalités politiques (mečtanija, selon l'expression de Jakov) qui faisaient la grandeur de la révolution, étaient des projets orientés vers l'avenir. Elles étaient réellement débattues, avec la possibilité pour chacun de mettre le doigt sur les obstacles qu'il voyait et qui étaient encore à franchir pour réaliser ces projets. Pendant la révolution, la politique était considérée comme un moyen pour atteindre un but supérieur: l'édification du communisme (que chacun imaginait à sa façon). Dès lors, si la fin justifiait les moyens ${ }^{46}$, cette relation entre fin et moyens a subi des changements décisifs sous le règne de Stalin, dans la mesure où le côté « fin » a été complètement éliminé, dès que l'on a tout simplement «fixé » (zafiksirovali, c'est-à-dire enregistré comme un fait) l'aboutissement d'un plan, ou bien même la réalisation du socialisme, comme Spunde l'avait déjà affirmé en 1936 à propos de la Constitution stalinienne. Les paroles de Stalin sur « la vie » qui « est devenue meilleure et plus joyeuse » représentent non seulement une attaque contre la réalité des faits l'affirmation n'était juste qu'en comparaison d'avec 1932, comme Spunde le constate dans sa lettre à Jakov de 1936 - , mais aussi un attentat contre l'utopie révolutionnaire : si on est déjà arrivé au bout, il n'y a plus rien à atteindre, ni même à attendre. Les moyens de la politique se sont émancipés de leurs fins. Alors que, pendant la guerre civile, on justifiait la terreur rouge comme inévitable pour se maintenir au pouvoir et poursuivre le grand projet de la libération de l'humanité, la terreur des années 1930 n'existe tout simplement pas dans les discours officiels. Elle est tabouisée. Les seuls terroristes sont les prétendus « ennemis du peuple».

Les appels au marxisme scientifique et même à l'instance des lois historiques (zakonomernost') - malgré les limites qu'elles imposent à la conceptualisation de l'enjeu de l'agir humain - sont donc en premier lieu des tentatives de rétablir les faits (fakty). C'est dire qu'ils se réfèrent à un autre niveau que celui des modèles interprétatifs, un niveau que les discours invoquent constamment pour se justifier contre n'importe quelle contestation ou critique qui recourt aux faits. La tentative de reconstituer le monde réel contre la «fiction stalinienne » explique l'acharnement avec lequel Spunde - et un grand nombre d'autres vieux bolcheviks, bien que souvent moins consciemment $-\mathrm{s}$ 'attellent à la rédaction de leurs Mémoires sur l'époque révolutionnaire, époque particulièrement déformée et falsifiée par «tous les Précis d'histoire». Ainsi, dans une des introductions à ses Mémoires, Spunde s'assigne-t-il deux tâches : donner l'évaluation d'une époque, dont il fut (et est encore) témoin et acteur et qui a contribué à former sa conception du monde depuis l'enfance, et « aider la prochaine génération à rétablir les traits (vosstanovit'

46. Cette logique heurterait les kantiens, mais n'oublions pas qu'elle n'est pas étrangère aux stratégies justificatives dans les débats politiques occidentaux où, tout au contraire, elle jouit actuellement d'une vogue particulière. 
oblik) des acteurs et créateurs des événements révolutionnaires ${ }^{47}$. Le second objectif est donc de réhabiliter les révolutionnaires (ou tout au moins leur mémoire), alors qu'ils sont calomniés et discrédités par toute la représentation officielle (stigmatisée comme mensongère).

2. Cependant, cette motivation tout à fait consciente de reconstitution des faits historiques n'explique pas vraiment que l'on trouve dans les archives personnelles de Spunde (comme dans celles d'un grand nombre d'autres vieux bolcheviks qui ont survécu aux purges) tant de versions de Mémoires sur l'époque révolutionnaire qui, généralement,s'interrompent dans le courant des années 1920. S’il ne s'agissait que d'une contestation de la déformation des faits, il aurait été non moins important d'écrire sur la manipulation des statistiques dans les années 1930, la falsification des bilans des plans quinquennaux par exemple. Trop pris à écrire et réécrire à maintes reprises sur l'époque de la révolution, Spunde n'arrive pas à mener ses Mémoires jusqu'au présent, contrairement à son projet. Pourquoi donc cette éternelle réécriture ? Lui était-il trop difficile de reconnaître sa propre responsabilité dans la mise en place de la collectivisation ? À en juger par les récits sur la révolution et la première moitié des années 1920, il était tout à fait capable d'analyser et de mettre en cause ses décisions et d'admettre ses erreurs.

Notre hypothèse est autre. Il y a dans cette expérience vécue de la révolution comme un trésor perdu que Spunde et les autres révolutionnaires n'arrivent pas à découvrir, mais qui les fait toujours revenir à la révolution, tant ils sentent qu'il y a encore quelque chose, peut-être l'essentiel, qu'ils n'ont pas encore saisi, qu'ils ne sont pas encore parvenus à nommer pour s'en assurer et éventuellement pour le transmettre à la génération suivante. C'est ce qui a donné sens à leur vie, qui les a dotés d'une dignité humaine particulière et ce qu'ils ont perdu en quelque sorte par la suite. Dans ses Mémoires, Spunde revient toujours sur le démocratisme profond, l'initiative et l'activité spontanée « d'en bas » (snizu), et avant tout sur les rapports humains (vzaimootnoščenija) qui, tels qu'on les a vécus à l'époque révolutionnaire, auraient en fait déjà été la réalisation d'un grand point du « programme social » de la révolution, en dépit d'une économie en ruines ${ }^{48}$ ! Dans les soviets il reconnaît la forme organisationnelle pour assurer le démocratisme, le contrôle du gouvernement et l'initiative autonome à tous les niveaux. Il souligne que, sur la base des soviets, on voulait réaliser ce que la Commune de Paris avait proclamé49. Voilà pourquoi il se montre particulièrement préoccupé par le rôle des soviets « après la victoire ». Il ne se contente pas d'attribuer la responsabilité de la quasi-liquidation des soviets au seul règne de Stalin. S'il décèle aussi dans les premières années du pouvoir soviétique « les points faibles qui ont fait s'effondrer tout le bâtiment », c'est que, tout en mettant en évidence les ruptures entre révolution et stalinisme, il

47. GARF, f. R-9665, op.1, d. 203,1.1.

48. GARF, f. R-9665, op.1, d. 205, 1.61. Cf. aussi le témoignage de Jakov sur son père et l'importance qu'il a accordée aux rapports humains (GARF, f. R-9665, op.1, d. 212,1.1-2).

49. GARF, f. R-9665, op.1, d. 205,1.49. 
reste sensible aux continuités. C'est ainsi qu'il évoque avec regret son vote pour la direction unique :

J'ai voté pour, mais avec un sentiment extrêmement pénible et avec une inquiétude poignante, parce que je me suis demandé si elle [la résolution] se révélerait compatible avec un véritable contrôle des couches inférieures sur les organes de pouvoir $^{50}$.

On peut donc discerner dans son récit quelque chose qui s'apparente à une expérience pluraliste. Il n'en retombe pas moins toujours dans une terminologie collectiviste de «masses populaires », de «camaraderie», qui brouille plus qu'elle n'éclaircit, parce que ces termes correspondent à une autre expérience forte, celle de la guerre civile, qui a forgé des armées et soudé les combattants en camarades (spajanie). Ces rapports humains sont extrêmement forts dans la mesure où les participants voient ensemble la mort de près, se sauvent mutuellement la vie, ce qui crée une proximité particulière ${ }^{51}$.

Mais il demeure crucial de démêler conceptuellement le chaos des diverses expériences de la révolution que Spunde et les autres vieux bolcheviks ont vécues. Il faut notamment distinguer entre rapports humains muets du combat, qui feront l'objet de la commémoration officielle de la révolution ${ }^{52}$, et les rapports langagiers de la délibération des affaires communes dans les innombrables espaces publics créés pendant la révolution. Dans les deux cas, les acteurs sont unis pour défendre un bien commun projeté dans l'avenir : l'édification du socialisme/communisme. Sous le règne de la violence muette du combat, le bien commun est toujours devant et, dans l'engagement pour le bien, les regards sont orientés dans ce sens : en avant (surtout vers l'ennemi qu'il faut vaincre). C'est dire que, côte à côte, les acteurs ne se regardent pas. Tout diffère dans la délibération des affaires communes dans l'espace public. Là, par la vertu de la communication, le bien, rendu présent, se situe entre les acteurs comme « intérêt = inter esse » (ce qui est entre) : par conséquent, en envisageant le bien commun, les acteurs se regardent aussi mutuellement. Ils sont en même temps égaux parce que liés par le respect mutuel pour l'authenticité de l'engagement de l'autre, et distincts parce que séparés par leurs perspectives, les positionnements de chacun vis-à-vis de ce bien entre eux. C'est la dynamique, la spontanéité de l'échange pluraliste des points de vue sur le bien commun qui engendre la formation des opinions, par essence subjectives, qui constituent par là la subjectivité des acteurs. Subjectivité prend ici un double sens : 1. celui de la

50. GARF, f. R-9665, op. 1, d. 205, 1. 49. Il s'interroge aussi longuement sur le traitement des membres des autres partis révolutionnaires après le soulèvement d'Octobre.

51. Sur ce genre d'expérience des masses et sa fascination, sur l'effacement de l'identité individuelle dans une sorte d'identité collective, cf. Elias Canetti, Masse et puissance, P. : Gallimard, 1986.

52. C'est bien la guerre civile qui est au centre de la commémoration, identifiée à la révolution même. Voir la littérature, les films, les musées, etc., mais aussi les défilés et célébrations qui recréent toute la formation de l'armée et évoquent consciemment cette expérience de gens soudés dans une masse. 
référence à une objectivité, le monde commun des faits (objectivité plus tard contestée par la fiction stalinienne et défendue avec tant de véhémence par Spunde dans son projet historiographique) ; 2. celui d'une distinction des acteurs les uns des autres ${ }^{53}$.

Mais quel est donc l'enjeu de cet échange en public ? En quoi diffère-t-il de la communication dans des cadres de validation restreints, ces espaces de proximité où - nous l'avons vu dans les échanges dans le cercle de la famille - nous pouvons aussi, entre autres, retracer des prises de position ? C'est que le soi s'amplifie par l'effet même de la grandeur et de la généralité du bien que l'on poursuit ${ }^{54}$, bien de la classe ouvrière, du pays, de l'humanité même. Mais qui peut assurer qu'il s'agit vraiment d'un bien commun? Sur le plan de l'expérience de soi, le bien est d'autant plus grand et commun qu'il y a de personnes qui s'engagent. Si peu de gens entrent en interaction, leurs préoccupations risquent fort de n'être que restreintes, bien plus mesquines qu'ils ne se l'imaginent. La grandeur du bien implique donc une multitude de gens qui s'engagent, prennent position et se font une opinion par rapport à ce bien. D'où le rôle essentiel de la persuasion dans les espaces publics, la capacité de rassembler derrière soi les opinions d'autres acteurs. La dynamique de regroupement et de ralliement des opinions dans un engagement authentique de nombreux acteurs constitue le phénomène du pouvoir, d'autant plus que c'est cette dynamique et cette large participation qui sont les seuls indicateurs de l'ampleur et du caractère commun du bien. Grand est celui qui sait au mieux représenter et défendre le bien. Cette conception d'un pouvoir dynamique, qui repose sur la pluralité et la réciprocité des acteurs, a été excellemment décrite par Hannah Arendt ${ }^{55}$. C'est cette expérience de pouvoir qui est en jeu dans la révolution, qui s'établit dans les soviets, et qu'il faut clairement distinguer de la domination, où le langage est dégradé en moyen de commandement et où la réciprocité de l'expérience et l'équilibre fragile d'engagements authentiques mutuels sont abolis - au même titre que l'expérience unique de soi ${ }^{56}$.

53. Cf. Hannah Arendt, La condition de l'homme moderne, passim pour une conceptualisation de l'agir politique.

54. Pour l'élucidation théorique des questions autour du rapport entre différents régimes d'engagement et de critique du familier au public, cf. Laurent Thévenot : «L'action comme engagement », in J.-M. Barbier, éd., L'analyse de la singularité de l'action, P. : Presses universitaires de France, 2000, p. 213-238 ; "Le régime de familiarité ; des choses en personnes », Genèses, 17, septembre 1994,p. 72-101; et surtout L'action au pluriel.

55. Cf. Hannah Arendt, On violence, New York - London-Harcourt : Barce Javanovich, 1970, où elle fait la distinction entre, d'une part, les rapports de domination basés sur la violence, réelle ou latente et, d'autre part, les rapports de pouvoir basés sur une équité entre les actants.

56. Les conflits entre l'opposition trotskiste de 1923-1924 et le triumvirat mettent très bien en relief l'opposition entre ces différentes expériences. Cf. Preobraženskij, «O našem vnutripartijnom položenii » [De notre situation à l'intérieur du parti], Pravda du 28.11.1923, et surtout la réponse de Stalin : «O zadačah partii » [Des devoirs du parti], Pravda du 2.12.23. Trockij, un des plus grands orateurs, qui se sentait comme « un poisson dans l'eau » dans les grands débats publics (et ceci dès la révolution de 1905), se trouve particulièrement en porte-à-faux à l'issue de la révolution et de la guerre civile. Il avait pris goût à la domination en tant que commandant en chef de l'Armée rouge, et voulut appliquer à la vie civile en 1920 son expérience de stratège. 
L'expérience de soi dans cette dynamique interactive de la révolution était particulièrement forte pour les leaders dans les débats, pour les grands orateurs et porteparole qui réussissaient à persuader les autres. Ce n'est que dans l'interaction avec les autres qu'ils pouvaient s'assurer de la grandeur du bien qu'ils poursuivaient. Mais dans la mesure où le foisonnement d'innombrables soviets du niveau local jusqu'au niveau public a créé une infinité de ces espaces publics, cette expérience de grandeur était fondatrice pour de nombreuses personnes engagées à travers le pays tout entier, particulièrement pour les vieux bolcheviks, qui étaient parmi les plus engagés. Même s'ils étaient loin de disposer d'un concept pour définir leur expérience révolutionnaire, ils avaient vécu cette expérience : de l'obscurité de l'espace du familier (ou du jour douteux de la conspiration au sein du parti) ils entrent, avec la révolution, dans la clarté et la lumière de l'espace public où ils s'assurent de la grandeur et du caractère commun des biens qu'ils défendent. En arrière-plan de cette expérience, il n'est pas étonnant que Spunde, avec ses propos universels, tende à sortir du cadre de la famille, par le biais de ses Mémoires qu'il veut faire circuler pour toucher « la jeune génération » et ainsi rétablir un espace public pour s'assurer de la grandeur et du caractère commun des biens pour lesquels il s'engage ${ }^{57}$. Visiblement, la nouvelle activité « des masses populaires » pendant la Seconde Guerre mondiale l'a encouragé dans ce sens ${ }^{58}$. Il est néanmoins évident, si l'on regarde le noyau de ses Mémoires, que cette tentative d'étendre la discussion vers un public se fonde essentiellement sur l'expérience révolutionnaire qu'il cherche à renouveler.

Sur ce fond, nous proposons une relecture des pratiques staliniennes, notamment des grands procès publics qui opèrent, à notre avis, sur des niveaux plus subtils et plus profonds que la crainte et la peur. C'est l'acte langagier seul qui rend possible la communication sur le bien commun. Avec l'articulation d'une opinion par rapport au bien, il révèle la personne unique, dans sa singularité. Dans cet échange pluraliste de la révolution que l'on peut entrevoir dans les récits de

C'était surtout l'illusion de la prévisibilité et du contrôle, l'idée que l'on pouvait maîtriser l'avenir qui était séduisante, et pas seulement pour Trockij. En revanche la dynamique de la discussion, et ses résultats inattendus, donnait l'impression d'un manque de contrôle qui menaçait l'avenir. Comme Spunde et Trockij, bien d'autres bolcheviks avaient connu des expériences révolutionnaires diverses à l'origine de perceptions de soi-même et des autres divergentes. Ces contradictions étaient d'autant plus difficiles à maîtriser que les acteurs ne disposaient pas alors d'un outillage conceptuel permettant de distinguer nettement la nature de ces différentes expériences.

57. Même si ce n'était que dans un cadre restreint, les Mémoires ont apparemment circulé, de sorte que nous pouvons y déceler un précurseur du samizdat et des pratiques des dissidents, qui ont finalement réussi à établir un « contre-public », comme c'était déjà l'intention de Spunde. L'activité des dissidents poursuit la même logique d'un bien commun qui s'appuie sur les idéaux révolutionnaires dans sa critique du régime au pouvoir.

58. Avec beaucoup d'espoir il observe les changements dans l'armée soviétique et se montre particulièrement attentif au mouvement des partisans où il semble remarquer une activité plus ou moins indépendante des instances de contrôle du commandement. 
Spunde, l'expérience de soi repose ainsi sur deux piliers qui sont systématiquement déniés par les pratiques staliniennes :

- la réalité du monde commun autour duquel les acteurs se regroupent et qui est l'objet de leurs engagements et paroles ;

- la reconnaissance de l'authenticité de l'engagement de l'autre, pour autant que c'est à partir de là que l'on oriente ses propres actions et engagements.

Nous ne prenons au sérieux les arguments de notre interlocuteur qu'autant que nous ne le soupçonnons pas constamment d'hypocrisie, c'est-à-dire que nous ne le soupçonnons pas de seulement feindre une préoccupation par le bien qui nous concerne tous deux et de poursuivre en réalité des desseins cachés. Si dans un différend avec autrui nous nous engageons totalement pour une cause, et qu'il s'avère par la suite que l'autre a seulement simulé un intérêt, alors nous sentons mis à nu car l'objet de notre débat n'est plus un bien commun. Notre engagement langagier et notre dévoilement concomitant en tant que personne engagée sont plus que perdus dans le vide, ils deviennent ridicules. Et ce d'autant plus, si dans un espace public, la plupart des grands acteurs vis-à-vis desquels nous nous sommes engagé, personnages prétendument intègres et authentiquement engagés, se révèlent hypocrites ${ }^{59}$. Perception et expérience de soi dépendent de la perception par les autres. Une perception réciproque communiquée, au-delà des contenus, dans l'interaction langagière et la réalité de l'expérience - déjà fragile par sa réciprocité - est sapée par le dévoilement, à grande échelle, de l'hypocrisie et du crime, c'est-à-dire de l'inauthenticité des communicants. La chasse à ces hypocrites par excellence que sont les espions, à l'échelle de masse sape aussi cette expérience de soi en détruisant l'authenticité de l'engagement commun. Il ne suffisait cependant pas d'inculper les acteurs de corruption dans leurs fonctions au présent, de leur reprocher d'avoir progressivement dégénéré, séduits par la vie luxueuse et les offres des services de renseignements étrangers. Il fallait porter l'accusation d'hypocrisie jusqu'à la révolution même ${ }^{60}$ pour miner cette expérience pluraliste forte dont la réciprocité était constitutive pour la subjectivité des acteurs. Au-delà donc d'une tentative de casser les liens personnels et publics, surtout dans le passé révolutionnaire, c'était par là la personne elle-même que l'on voulait attaquer, dans la mesure où elle s'était seulement constituée comme telle, avec toute son assurance et son identité politique, par le biais de l'interaction, de l'agir ensemble.

Le seul moyen d'éradiquer une telle expérience dynamique (et incontrôlable) est d'y substituer la fiction. Voilà pourquoi Stalin fait réécrire l'histoire du parti, notamment l'histoire de la révolution, qu'il liquide non seulement les grands personnages, mais leur mémoire aussi... Cependant il ne pouvait pas le faire seul,

59. Voir les réflexions d'Hannah Arendt sur le masque et l'hypocrisie, Essai sur la révolution, P. : Gallimard, 1985.

60. Si l'on suit les procès-verbaux des trois grands procès de 1936-1938, on se rend vite compte que l'accusation d'hypocrisie est reportée de plus en plus loin dans le passé, pour finir par empoisonner la révolution elle-même. Ce n'était cependant pas totalement faux : un certain nombre de révolutionnaires, surtout au Parti bolchevik, avaient été agents de la police secrète tsariste (les plus connus étant Papon et Malinovskij). 
même la violence pure et dure est impuissante contre le pouvoir d'une mémoire partagée et constamment réactivée. Il fallait nier l'expérience communément, établir des espaces où le mensonge fut diffusé et amplifié par une multitude de gens, il fallait discuter et affirmer l'accusation d'hypocrisie dans toutes les réunions sur tous les niveaux du pays. Un éditorial dans la Pravda ou autres journaux n'était pas suffisant. Il fallait que les gens en parlent, qu'ils y participent, qu'il y ait de l'interaction, de l'échange langagier autour du mensonge, que tous affichent leur dédain pour les « salauds », les « agents fascistes », les « chiens enragés », etc. En ce sens Stalin a pris des leçons de l'expérience révolutionnaire dynamique à laquelle il a assisté dans l'ombre des grands orateurs qui savaient persuader. Il n'a pas simplement réprimé le débat et la mémoire de cette expérience pluraliste révolutionnaire, il l'a détournée pour mettre en place une réciprocité de la fiction, qui seule pouvait doter cette fiction d'un sens de « réalité ».

Là où il y a dynamique interactive autour du mensonge, il n'est pas toujours facile de rétablir même la pure facticité, comme Spunde veut le faire. Les éléments vraiment troublants des Mémoires de Spunde révèlent l'impact de la fiction stalinienne sur le sens de la réalité. Ainsi, visiblement, dans ses Mémoires des années 1940, Spunde croit, malgré toute sa lucidité, aux témoignages de Krestinskij durant le procès de 1938. Des trotskistes auraient pris contact avec le commandement militaire prussien en 1919 afin d'obtenir du soutien dans la lutte de pouvoir (c'est-à-dire de domination) au sein du parti ${ }^{61}$. Nous assistons à la manière dont il révise ses propres impressions de l'époque : il affirme qu'alors il ne lui serait jamais venu à l'esprit que les discussions n'étaient pas véritablement centrées autour de la cause, du contenu, que derrière les mots des délégués il y avait une arrière-pensée qui était la vraie motivation de leurs actions, celle de s'emparer de la direction du parti. Nous notons ici, sur la base des aveux dans le procès exemplaire, le doute qui s'installe, doute par rapport à l'authenticité des motivations des interlocuteurs. Chez lui ce sont particulièrement ses entretiens personnels avec Krestinskij qui subissent une telle réévaluation. Il affirme que leurs rapports étaient toujours un peu formels et qu'il n'a jamais pu s'épancher (otvesti dušu) avec Krestinskij, comme il l'a pu avec Sosnovskij ou d'autres. Mais il ne voit là qu'une particularité du caractère de Krestinskij, sans que cela puisse amoindrir le respect qu'il porte au révolutionnaire. Maintenant, affirme-t-il, à la lumière des confessions de Krestinskij, je comprends mieux que je m'étais déjà trompé alors sur la sincérité des membres des fractions dans les débats sur le rôle des syndicats ${ }^{62}$.

Nous discernons désormais quel fut l'enjeu réel des procès politiques. Accuser d'anciens combattants bolcheviks non seulement d'être corrompus et d'être devenus criminels au présent, mais d'avoir été des agents de l'étranger ou de l'Ohrana, c'est-à-dire des hypocrites dès la révolution, c'était porter un coup au

61. Krestinskij était en fait le seul accusé qui ait tenté de s'opposer et de démentir ce qu'on lui reprochait. Or, après une pause apparemment non planifiée, il fut « ramené à la raison » et par la suite on obtint ses aveux «comme il fallait». Cf. Sudebnyj otčet po delu Antisovetskogo «Pravo-trockistskogo bloka ».

62. GARF, f. R-9665, op. 1, d. 205, 1. 52-55. 
cœur même de l'expérience qui, dans des cadres de proximité, était toujours potentiellement renouvelable, comme la dynamique des échanges au sein de la famille Kravčenko - Spunde l'a montré.

Université de Bielefeld

Centre Marc Bloch, Berlin

malte.griesse@uni-bielefeld.de 